\title{
Particle-resolved simulations of shock-induced flow through particle clouds at different Reynolds numbers
}

\author{
Andreas Nygård Osnes $\odot,{ }^{1,2, *}$ Magnus Vartdal $\odot,{ }^{2, \dagger}$ \\ Marianne Gjestvold Omang $\odot, 3,4, \ddagger$ and Bjørn Anders Pettersson Reif ${ }^{1, \S}$ \\ ${ }^{1}$ Department of Technology Systems, University of Oslo, P.O. Box 70 Kjeller, NO-2027 Kjeller, Norway \\ ${ }^{2}$ Norwegian Defence Research Establishment, P.O. Box 25, NO-2027 Kjeller, Norway \\ ${ }^{3}$ Norwegian Defence Estates Agency, P.O. Box 405 Sentrum, NO-0103 Oslo, Norway \\ ${ }^{4}$ Institute of Theoretical Astrophysics, University of Oslo, P.O. Box 1029 Blindern, NO-0315 Oslo, Norway
}

(Received 21 June 2019; published 27 January 2020)

\begin{abstract}
This study investigates the Reynolds-number dependence of shock-induced flow through stationary particle clouds at $10 \%$ volume fraction, using ensemble-averaged results from three-dimensional particle-resolved large eddy simulations. The advantage of using large eddy simulations to study this problem is that they capture the strong velocity shears and flow separation caused by the no-slip condition at the particle surfaces. The shock particle cloud interaction produces a reflected shock wave, whose strength increases with decreasing particle Reynolds number. This results in important changes to the flow field that enters the particle cloud. The results show an approximate proportionality between the mean flow velocity and the flow fluctuation magnitudes. Maximum particle drag forces are in excellent agreement with previous inviscid studies, and we complement these results with statistics of time-averaged particle forces as well as the variation of temporal oscillations. The results of this work provide a basis for development of improved simplified dispersed flow models.
\end{abstract}

DOI: 10.1103/PhysRevFluids.5.014305

\section{INTRODUCTION}

Interactions between shock waves and dense suspensions of particles occur in many engineering applications and industrial processes. Some examples are liquid and solid fuel engines [1-3], heterogeneous explosives [4], blast mitigation [5,6], and dust-explosion safety measures [7]. Natural occurrences of shock-wave particle cloud interactions include volcanic eruptions [8] and supernovae $[9,10]$.

The process of shock-wave particle cloud interaction has recently received attention in both experimental [11-13] and numerical studies [14-19]. Numerical studies have primarily utilized two different approaches. One approach is to solve an averaged form of the compressible NavierStokes equations. The model equations for the continuous phase are formulated to account for the volume and density of the dispersed phase and are most commonly solved with Eulerian descriptions. The dispersed phase can be represented with Eulerian or Lagrangian descriptions, and these models are therefore referred to as Eulerian-Eulerian or Eulerian-Lagrangian dispersed flow models. It is also possible to use Lagrangian descriptions for both phases, such as with smoothed

\footnotetext{
*Andreas-Nygard.Osnes@ffi.no

†Magnus.Vartdal@ffi.no

†m.g.omang@astro.uio.no

§.a.p.reif@its.uio.no
} 
particle hydrodynamics methods [20]. There are several problems with such simplified dispersed flow models, such as nonhyperbolic equation sets and erroneous particle dispersal predictions, as discussed in Lhuillier et al. [21] and Theofanous and Chang [19]. The second approach is to resolve the particles on the computational grid. This approach circumvents the problems of the simplified dispersed flow models, since the governing equations for the continuous phase are the standard compressible flow equations. This is the method utilized in this study. Particle-resolved simulations are valuable because they provide highly resolved data, in both space and time, which enables a comprehensive analysis. The primary limitation of such simulations is that only small-scale problems are computationally feasible.

There is a considerable body of work investigating shock-wave particle cloud interaction using particle-resolved inviscid simulations [22-27]. The number of studies that have included viscous effects in such simulations is, however, limited. Particle-resolved studies that have included viscous effects are, e.g., the two-dimensional simulations of Das et al. [28], Hosseinzadeh-Nik et al. [29], and Naiman and Knight [30] and the three-dimensional simulations of Osnes et al. [31] and Vartdal and Osnes [32]. Viscous effects have not been analyzed directly in experimental studies either. There is, however, a moderate range of particle Reynolds numbers, $\operatorname{Re}_{\mathrm{p}}$, where experimental results exist. Based on the shock-wave strength, the particle Reynolds numbers in Wagner et al. [13] are approximately $2650-4200$. The study of DeMauro et al. [11] spans $\operatorname{Re}_{\mathrm{p}} \approx 900-5700$, whereas Theofanous et al. [12] investigated configurations in the range $\operatorname{Re}_{\mathrm{p}} \approx 7000-70000$. Since these studies vary Reynolds number simultaneously with Mach number and ratio of layer thickness to particle diameter, isolating the Reynolds-number effects is difficult.

The Reynolds number is a measure of the relative importance of inertial to viscous forces within the flow. Viscosity has an effect on the flow field even at very high particle Reynolds numbers, as long as the flow remains in the continuum regime. In particular, viscous effects are responsible for flow separation behind particles. This is in fact an important phenomenon in shock-wave particle cloud interaction, as will be shown in this study.

This study considers particle Reynolds numbers in the range 500-10000. In incompressible, single-particle flows, this particle Reynolds number range spans three different flow regimes, by their definitions in Tiwari et al. [33]. These flow regimes are commonly labeled the vortex shedding regime $\left(270 \leqslant \operatorname{Re}_{\mathrm{p}} \leqslant 800\right)$, the separating vortex regime $\left(800<\operatorname{Re}_{\mathrm{p}} \leqslant 3000\right)$, and the subcritical regime $\left(3000<\mathrm{Re}_{\mathrm{p}}<3.4 \times 10^{5}\right)$. Sakamoto and Haniu [34] discuss the flow properties over this range and draw the following conclusions. In the vortex shedding regime, laminar vortices are shed periodically. Above $\mathrm{Re}_{\mathrm{p}}=800$, vortex tubes and vortex loops are formed in the vortex sheet and can interact with the large-scale laminar vortices, introducing irregularity in the vortex shedding. Above $\mathrm{Re}_{\mathrm{p}}=3000$, the vortex sheet becomes turbulent, and the turbulence intensity increases with $\mathrm{Re}_{\mathrm{p}}$. As a result of this, the Strouhal number, i.e., the nondimensional vortex shedding frequency, decreases with Reynolds number. At $\mathrm{Re}_{\mathrm{p}}=6000$ and above, the vortex sheet is fully turbulent, and the Strouhal number is approximately constant.

Compressible flows over isolated particles in this Reynolds number range have not received nearly as much attention. Nagata et al. [35,36] investigated $50 \leqslant \operatorname{Re}_{\mathrm{p}} \leqslant 1000$. Notably, the drag coefficient increases by more than a factor of two when the flow goes from subsonic to supersonic in this $\mathrm{Re}_{\mathrm{p}}$ range. The Mach number, Ma, also has a stabilizing effect on the flow, and Nagata et al. [36] found that at $\mathrm{Ma}=2$ the flow is stable to at least $\mathrm{Re}_{\mathrm{p}}=1000$.

It is not straightforward to apply these findings to shock-wave particle cloud interaction. Importantly, the incident flow becomes subsonic due to the generation of a planar, upstream-propagating, reflected shock $[11,13,37]$. The strength of this shock determines which flow conditions the particles are exposed to, and these flow conditions define a particle Reynolds number and a local Mach number that is not known a priori. These parameters are likely more appropriate for classification of the flow through the particle cloud after the initial shock-induced transient than the ones based on flow properties behind the incident shock. Additionally, the flow Mach number increases with downstream distance within the particle cloud [25,27,31] in a manner similar to that observed in 
Fanno flows [38]. The presence of nearby particles also affects the flow around each particle by changing the direction of the incoming flow, exposing it to particle wakes, etc.

As will be shown in this study, the particle Reynolds number affects the level of particle-scale fluctuations within the particle cloud. These fluctuations are defined here as the deviations from the volume-averaged velocity and need not be turbulent fluctuations; a laminar particle wake generates significant fluctuations. When the particle concentration increases, the impact of the wake-induced fluctuations also increases. In particular, particle wakes start to comprise a significant part of the volume within the cloud. This causes a problem for models based on volume averaging. The volumeaveraged velocity is shifted towards the average particle velocity, and as a result, it represents neither the flow in immediate proximity of each particle nor the freer flow between them. In the current work, we explore what this means for the fluctuation levels and particle drag coefficients.

The current study is concerned with how the particle Reynolds number affects shock-wave particle cloud interaction. To this end, we perform particle-resolved, three-dimensional, viscous simulations of the passage of a shock wave through a particle layer with volume fraction 0.1 . The particle positions are assumed to be fixed over the time frame considered. The particle Reynolds numbers in this work are 500, 2000, 5000, and 10000 . For the higher Reynolds numbers, the turbulent structures in the boundary layers around the particles and in their wakes are not fully resolved. However, the unresolved scales are unlikely to introduce more than minor alterations to the main results in this work.

We examine shock-wave attenuation and reflection, mean flow fields, and fluctuation statistics. We also explore the Reynolds number dependence of forces acting on the fixed particles, including maximal values, time-averages, and distributions of the drag coefficients. The results from the current study are important as a baseline for development of closures for simplified dispersed flow models, as well as the formulation of particle force models for shock-wave particle cloud interaction.

This paper is structured as follows. In Sec. II we describe the equations governing the flow through the particle cloud, the mathematical framework used in the flow analysis, and the computational method. Section III specifies the problem under investigation. The simulation results are presented and discussed in Sec. IV, and Sec. V contains concluding remarks.

\section{GOVERNING EQUATIONS AND COMPUTATIONAL APPROACH}

The motion of the fluid is governed by the compressible Navier-Stokes equations, which can be written

$$
\begin{gathered}
\partial_{t} \rho+\partial_{k}\left(\rho u_{k}\right)=0, \\
\partial_{t}\left(\rho u_{i}\right)+\partial_{k}\left(\rho u_{i} u_{k}\right)=-\partial_{i} p+\partial_{j} \sigma_{i j}, \\
\partial_{t}(\rho E)+\partial_{k}\left(\rho E u_{k}+p u_{k}\right)=\partial_{j}\left(\sigma_{i j} u_{i}\right)-\partial_{k}\left(\lambda \partial_{k} T\right),
\end{gathered}
$$

where $\rho(\mathbf{x}, t)$ is the mass density, in which $\mathbf{x}$ is the spatial coordinate vector and $t$ denotes time, $u(\mathbf{x}, t)$ is the velocity, $p(\mathbf{x}, t)$ is the pressure, $\sigma_{i j}(\mathbf{x}, t)=\mu\left(\partial_{j} u_{i}+\partial_{i} u_{j}-2 \partial_{k} u_{k} \delta_{i j} / 3\right)$ is the viscous stress tensor, $\mu(\mathbf{x}, t)$ is the dynamic viscosity, $E(\mathbf{x}, t)=\rho e+0.5 \rho u_{k} u_{k}$ is the total energy per unit volume, $e(\mathbf{x}, t)$ is the internal energy per unit mass, $\lambda(\mathbf{x}, t)$ is the thermal conductivity, and $T(\mathbf{x}, t)$ is the temperature. Here $\partial_{t}$ denotes partial differentiation with respect to time, and $\partial_{i, j, k}$ denotes partial differentiation with respect to space. The subscripts denote tensor components, and repeated indices imply summation from 1 to 3 . For convenience, we will also use $u, v, w$ without subscripts to denote the velocity components in the $x, y$, and $z$ directions, respectively. Internal energy, pressure, and density are related by the ideal gas equation of state, with $\gamma=1.4$. Temperature and internal energy are related by a constant specific heat capacity. We assume that the viscosity varies with temperature as $\mu(\mathbf{x}, t)=\mu^{0}\left(T / T^{0}\right)^{0.76}$, where $\mu^{0}$ is the viscosity at $T=T^{0}$. We further relate the thermal diffusivity to the viscosity through a constant Prandtl number of 0.7 . 
The analysis in this work is based on the volume-averaged momentum conservation equations. A discussion of volume averaging for flows containing particles can be found in, e.g., Crowe et al. [39] and Schwarzkopf and Horwitz [40]. Since the configuration is statistically one-dimensional in space, only the streamwise momentum equation is relevant. It can be expressed as

$$
\partial_{t}\left(\alpha\langle\rho\rangle \tilde{u}_{1}\right)+\partial_{x}\left(\alpha\langle\rho\rangle \tilde{u}_{1} \tilde{u}_{1}+\alpha\langle p\rangle\right)=\partial_{x}\left(\alpha\langle\sigma\rangle_{11}\right)-\partial_{x}\left(\alpha\langle\rho\rangle \tilde{R}_{11}\right)+\frac{1}{V} \int_{S} p n_{1} d S-\frac{1}{V} \int_{S} \sigma_{1 k} n_{k} d S,
$$

where $\alpha$ is the gas volume fraction, $\tilde{R}_{i j}(\mathbf{x}, t)=\widetilde{u_{i}^{\prime \prime} u_{j}^{\prime \prime}}$ is the velocity fluctuation correlation tensor, $V$ denotes the averaging volume, $n$ is the normal vector pointing into the continuous phase, and $S$ denotes the continuous phase boundary. Here $\langle\cdot\rangle$ denotes a phase average, $\sim$ denotes a Favre average, and fluctuations from the Favre average are denoted .". Furthermore, we denote the fluctuating kinetic energy per unit volume, $\langle\rho\rangle \tilde{R}_{i i} / 2$, by $k$, and mean kinetic energy per unit volume by $K$. The particle volume fraction is denoted by $\alpha_{\mathrm{p}}$.

In the analysis of the computational results, the temporal evolution is discussed in terms of a timescale related to the passage of the shock through the particle layer. This timescale is

$$
\tau_{L}=L / u_{\mathrm{s}}
$$

where $u_{\mathrm{s}}$ is the speed of the incident shock wave and $L$ is the length of the particle cloud. A second timescale we will use is related to the passage of the shock over a single particle,

$$
\tau_{\mathrm{p}}=D_{\mathrm{p}} / u_{\mathrm{s}}
$$

where $D_{\mathrm{p}}$ is the particle diameter. Furthermore, we use $t_{0}$ to denote the time point when the shock wave is at $x=0$.

The particle drag coefficient will be used in the discussion of particle forces. It is

$$
C_{\mathrm{D}}=\frac{\int_{S_{\mathrm{p}}}\left(p n_{1}-\sigma_{1 k} n_{k}\right) d S}{0.5\langle\rho\rangle \tilde{u}_{1}^{2} A_{\mathrm{p}}}
$$

where $S_{\mathrm{p}}$ is the particle surface and $A_{\mathrm{p}}$ is the projected area of the particle in the flow direction.

\section{Computational method}

The present simulations are conducted using the compressible flow solver "CharLES" [41], developed by Cascade Technologies. The compressible, filtered, Navier-Stokes equations are solved on an unstructured Voronoi-based grid [42]. CharLES is a finite-volume large-eddy simulation code. It computes fluxes between control volumes based on an entropy-stable modified Lax-Friedrich flux. A more detailed discussion of the entropy-stable scheme can be found in Masquelet et al. [43]. A third-order explicit Runge-Kutta scheme is applied to advance the solution in time. The unresolved scales are modeled with the Vreman model [44].

\section{SETUP AND COMPUTATIONAL GRID}

We consider the interaction between a planar shock wave and a cloud of particles at different $\mathrm{Re}_{\mathrm{p}}$. Figure 1 shows a sketch of the computational setup. The computational domain is $-0.9 L \leqslant x \leqslant 2 L$, $0 \leqslant y \leqslant L_{y}$, and $0 \leqslant z \leqslant L_{y}$, where $L_{y}=8 \sqrt[3]{4} D_{\mathrm{p}}$. The particles are located within $0 \leqslant x \leqslant L$, and the particle volume fraction is 0.1 . In all configurations, the length of the particle layer and the particle diameter are related by $L=12 \sqrt[3]{16} D_{\mathrm{p}}$. The particles are drawn randomly within this range, and we require that no particle boundary extends outside $0 \leqslant x \leqslant L$. The minimum distance between each particle is set to roughly $0.05 D_{\mathrm{p}}$. The shock-wave position is initially $-0.1 L$ and is indicated by the thick line in the figure. Its direction of propagation is from left to right.

The computational grid is an unstructured Voronoi-based grid, which is body fitted to the particles. We use three different refinement levels, as indicated by the Roman numerals in Fig. 1. 


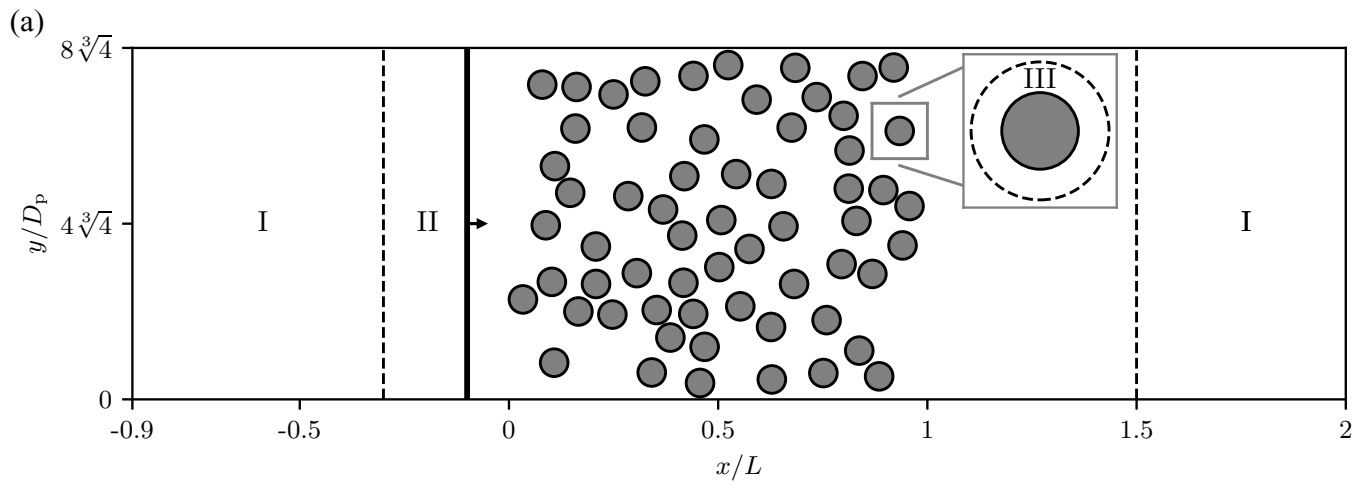

(b)

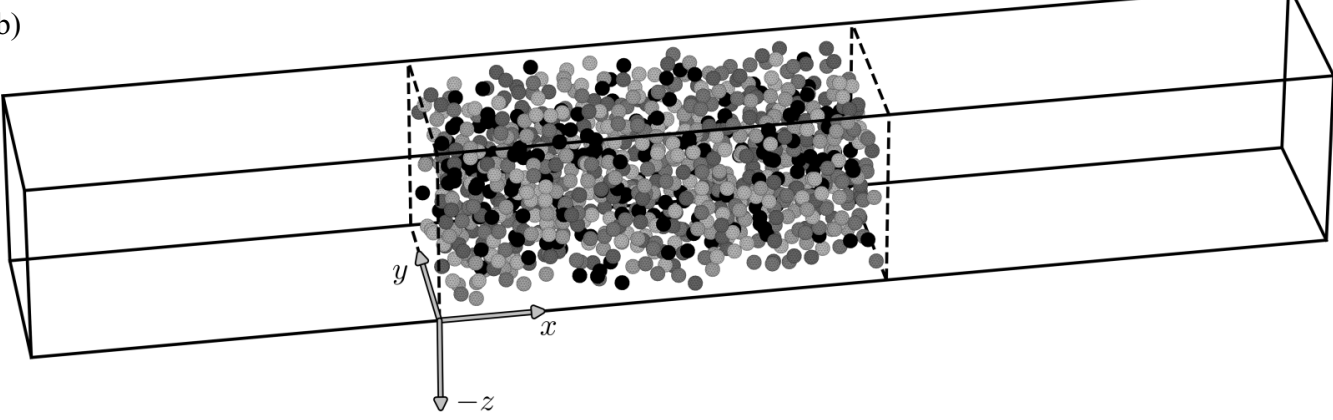

FIG. 1. Sketch of the computational setup and domain. The particle layer is located between $0 \leqslant x \leqslant L$, where the particle volume fraction is $\alpha_{\mathrm{p}}=0.1$. In (a), the thick solid line indicates the initial position of the shock wave, and the arrow shows its propagation direction. The Roman numerals indicate the level of grid refinement, where the control volumes in region I are eight times larger than those in region II, and those in region II are again eight times larger than those in region III (by volume). The dashed lines indicate the boundaries between the refinement levels. Note that the vertical axis has been stretched for illustration purposes. In (b) a three-dimensional view is shown, where the particle positions correspond to those used in one of the simulations.

Refinement level I has control volumes eight times larger (by volume) than level II, and similarly for levels II and III. The length scale of the smallest control volumes (level III) is roughly $0.036 D_{\mathrm{p}}$. The level III regions extend approximately $D_{\mathrm{p}} / 2$ out from each particle surface. Due to the high particle volume fraction most of the region $0 \leqslant x \leqslant L$ contains level III control volumes. The total number of control volumes is roughly $10^{8}$. The control volume sizes are similar to those used in Osnes et al. [31,45]. Those studies investigated grid sensitivity based on the grid convergence of the particle forces, resolution of viscous shear length scales, and convergence of spanwise velocity fluctuation correlations.

This study investigates the effect of particle Reynolds number over the range 500-10000 on the shock-induced flow through particle clouds. Specifically, we consider particle Reynolds numbers 500, 2000, 5000, and 10000 . The particle Reynolds number is defined as

$$
\operatorname{Re}_{\mathrm{p}}=\frac{\rho u D_{\mathrm{p}}}{\mu}
$$

Throughout this paper, the Reynolds number is based on flow properties behind the incident shock wave, unless otherwise specified. The subscript "IS" will be used to denote postshock values for the other flow variables. The Reynolds number is systematically varied by altering the viscosity, rather 
than varying the particle diameter or the shock-wave strength. This enables the use of the same computational grid for different Reynolds numbers.

The initial conditions consist of two homogeneous spatial regions, which are separated by the shock wave at $x=-0.1 L$. The preshock conditions are chosen to correspond to air at atmospheric conditions: $\rho^{0}=1.2048 \mathrm{~kg} / \mathrm{m}^{3}, u^{0}=0 \mathrm{~m} / \mathrm{s}$, and $p^{0}=1.01325 \times 10^{5} \mathrm{~Pa}$. The viscosity $\mu^{0}$, is varied to obtain the desired particle Reynolds number. At $\operatorname{Re}_{\mathrm{p}}=5000$, this corresponds approximately to the viscosity of air at standard atmospheric conditions $\left(\mu^{0} \approx 1.8 \times 10^{-5} \mathrm{~kg} / \mathrm{ms}\right)$. The Mach number of the incident shock wave is 2.6 , and behind it is a homogeneous flow with the postshock state, which is $\rho_{\mathrm{IS}}=4.16 \mathrm{~kg} / \mathrm{m}^{3}, u_{\mathrm{IS}}=633 \mathrm{~m} / \mathrm{s}$, and $p_{\mathrm{IS}}=782 \mathrm{kPa}$. At the $x=-0.9 L$ boundary, we apply constant inflow conditions, corresponding to the postshock state. At $x=2 L$, we use a zero-gradient outlet. The spanwise boundaries are periodic. The spanwise domain lengths are both roughly 13 particle diameters, and this should be sufficient to hinder any periodic artifacts. The simulations are run for a total time of $3.75 \tau_{L}$.

Flow field statistics are obtained by averaging flow variables over predetermined volumes. In the current work, we utilize volumes spanning the computational domain in the $y$ and $z$ directions, with a streamwise length of $L / 60 \approx 0.5 D_{\mathrm{p}}$.

A large number of particles is required in the spanwise directions to achieve well-resolved statistics of the time-dependent flow field. Since only a limited number of particles is feasible to include in a single simulation, we perform an ensemble of simulations at each $\mathrm{Re}_{\mathrm{p}}$. The gas-phase variables are presented in terms of volume-averaged quantities. The volume averages from the simulations are averaged over the ensemble of simulations, so that, for example,

$$
\langle\rho\rangle(\mathbf{x}, t)=\frac{1}{N_{\text {sim }}} \sum_{i=0}^{N_{\text {sim }}} \frac{1}{V^{i}(x)} \sum_{j=0}^{N_{\mathrm{CV}}^{i}(x)} \rho^{j}(t) V_{\mathrm{CV}}^{j},
$$

where $N_{\text {sim }}$ is the number of simulations (10 in this study), $N_{\mathrm{CV}}$ is the number of control volumes within the averaging volume $V(x)$, and superscripts denote quantities belonging to simulation $i$ or control volume $j$. Fluctuation correlations are computed implicitly as the difference between the products of the averages and the averages of the products. Thus, the velocity correlations are

$$
\widetilde{u_{i}^{\prime \prime} u_{j}^{\prime \prime}}=\widetilde{u_{i} u_{j}}-\tilde{u}_{i} \tilde{u}_{j} \text {. }
$$

We consider only statistics up to second-order moments, and we therefore use the convergence of the fluctuating kinetic energy, $k$, to estimate the required ensemble size. We find that the tenth simulation changes the statistics of $k$ by about $0.5 \%$ on average. We therefore consider an ensemble of 10 simulations at each $\operatorname{Re}_{\mathrm{p}}$ sufficient for the current analysis.

\section{RESULTS}

This section presents the simulation results. Section IV A provides a brief overview of the flow phenomena occurring when the shock wave passes through and interacts with the particle cloud. Section IV B examines the attenuation of the main shock wave and the strength of the reflected shock wave. Section IV C discusses mean fields and fluctuations in the gas phase. Finally, Sec. IV D presents particle drag coefficient trends and distributions.

\section{A. Flow description}

The incident shock impacts on the particle cloud at time $t_{0}$ and starts propagating through it. Each particle induces a shock-wave reflection, and at the upstream edge, these bow shocks coalesce into a planar reflected shock wave. The primary shock wave is continuously weakened as it propagates through the particle cloud. However, the length of the particle layer is insufficient to completely attenuate the shock wave, and therefore a transmitted shock emerges from the downstream cloud edge. The shock-induced flow is strongly unsteady, as can be seen in Fig. 2, which shows contours 


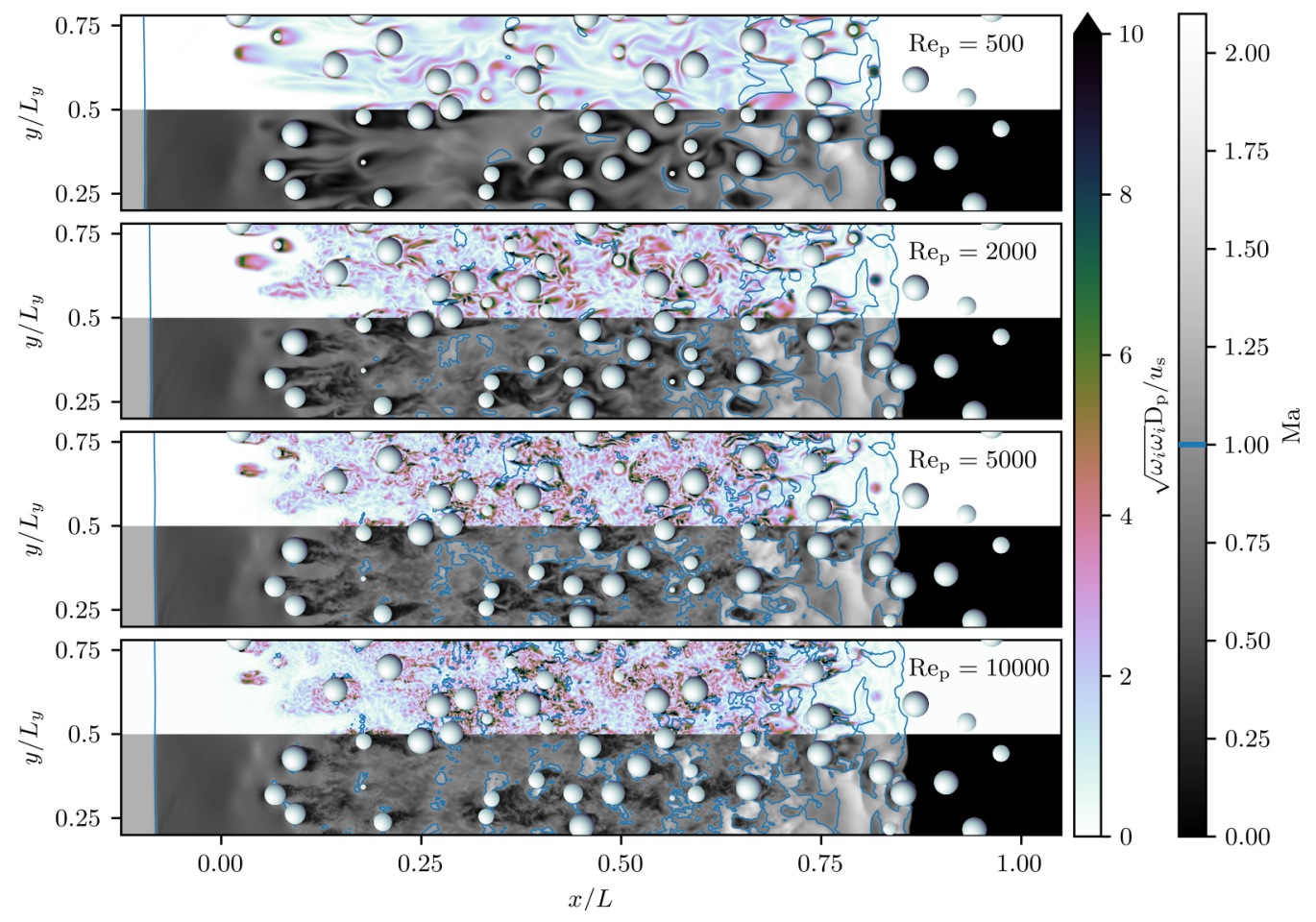

FIG. 2. Vorticity magnitude (top half of each contour plot) and local Mach number (bottom half) in a cut plane at $z=L_{y} / 2$ for different Reynolds numbers at $\left(t-t_{0}\right) / \tau_{L} \approx 0.83$. The highlighted contour is Ma $=1$ and separates regions of subsonic and supersonic flow. Note that the vorticity contours have been capped at $\sqrt{\omega_{i} \omega_{i}} D_{\mathrm{p}} / u_{\mathrm{s}}=10$. The vorticity magnitude in the small black regions exceeds this level, and the maximal value increases with $\mathrm{Re}_{\mathrm{p}}$. Only a part of the spanwise domain is shown.

of vorticity magnitude, $\sqrt{\omega_{i} \omega_{i}}$, and local Mach number, at $\left(t-t_{0}\right) / \tau_{L} \approx 0.83$ for the different Reynolds numbers. Behind the primary shock wave, there are large regions of supersonic flow between the particles. The velocity within the cloud decays over time, and the local supersonic regions become smaller. At high Reynolds number, however, the flow acceleration around the particles is strong enough to sustain localized supersonic flow regions, even at late times.

Particle wakes develop quickly after each particle interacts with the shock wave. When the shock wave has propagated a few particle diameters downstream of each particle, the particle wake can clearly be identified in both the vorticity and Mach number fields. Within the wake, the flow velocity is low and the flow is subsonic. The wakes are longer and more coherent at $\mathrm{Re}_{\mathrm{p}}=500$ than they are at the higher Reynolds numbers. In addition, the wakes are delineated by levels of high vorticity for $\operatorname{Re}_{\mathrm{p}}=500$. At higher Reynolds numbers, vorticity is still present within these shear layers, but the wakes are not as clearly delineated by vorticity because the vorticity levels are relatively high throughout large parts of the cloud. Vorticity is initially generated by baroclinic torque behind the shock wave, and later in the boundary layers around, and in the wakes of, the particles. Subsequently, vorticity is amplified by vortex stretching, and this term is about a magnitude larger than both baroclinic torque and viscous vorticity production. The high levels of vorticity indicate that the flow becomes more chaotic, or turbulent, with higher Reynolds numbers, but the largest velocity differences are still caused by the flow separation behind each particle, as indicated by the Mach number contours in Fig. 2. This motivates a detailed analysis of the flow fluctuations, in order to identify which microscale flow effects are dynamically important for shock-wave particle cloud interaction. 

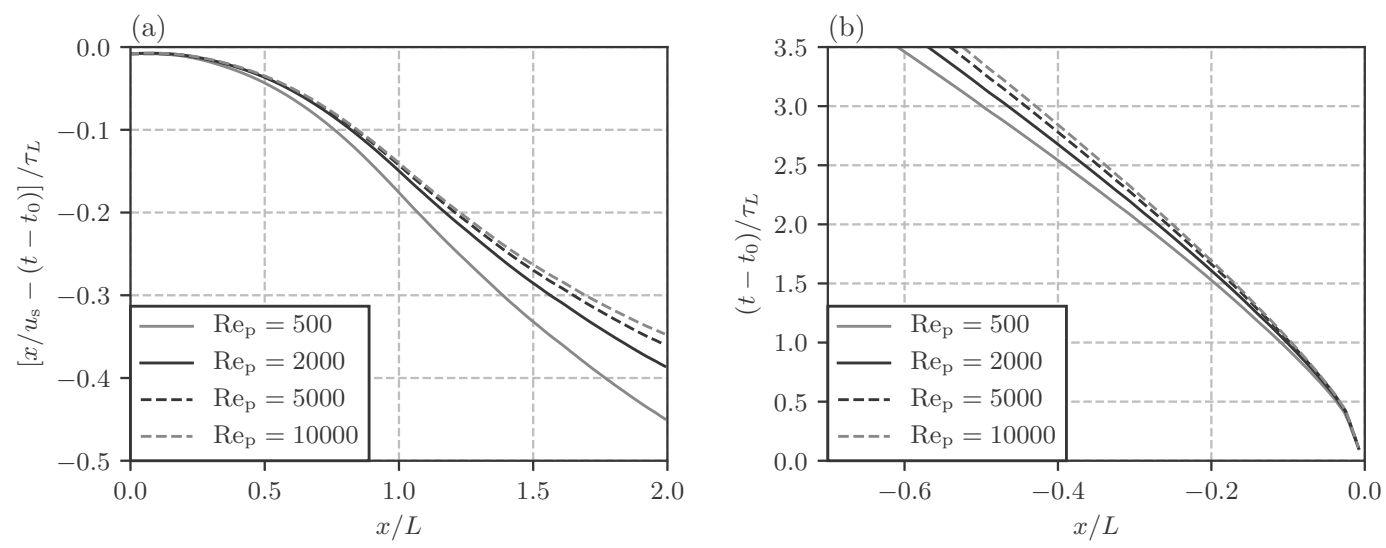

FIG. 3. (a) Main shock and (b) reflected shock position as a function of time for different $\operatorname{Re}_{\mathrm{p}}$. As opposed to the reflected shock, the variation in main shock position with $\mathrm{Re}_{\mathrm{p}}$ is small and is therefore plotted as the deviation from the trajectory of a hypothetical $\mathrm{Ma}=2.6$ shock wave with no attenuation.

\section{B. Shock-wave attenuation and reflection}

Figure 3 shows the main shock position as a function of time. The results are plotted as the deviation from the hypothetical trajectory of a shock wave with constant Mach number 2.6. This deviation is due to shock-wave attenuation caused by the presence of the particle cloud. Both shockwave reflection and particle drag contribute to the attenuation. The arrival time at a given location is delayed for lower Reynolds numbers.

If there is to be a considerable effect of $\mathrm{Re}_{\mathrm{p}}$ on the shock-wave attenuation, the impulse from viscous forces must be non-negligible on a timescale comparable to the passage of the shock wave over a particle. Viscous forces are caused by the no-slip condition at the particle surface, which sets up a strong velocity gradient around the particle. An estimation of the importance of viscous forces on the shock-wave attenuation can be obtained by comparing viscous and pressure forces acting on the particles during the time when the shock interacts with them. The average ratio of the viscous to pressure forces on the particles during the shock-particle interaction is given in Table I. It can be seen that for the lower Reynolds numbers, the viscous forces are appreciable even during the initial phase.

The Mach number of the transmitted shock wave, based on the average transmitted shock-wave speed over $L \leqslant x \leqslant 2 L$, is also given in Table I. The average transmitted shock speed is based on the shock arrival time at $x=L$ and $x=2 L$, where the arrival time is defined as the first instant where the volume-averaged pressure exceeds 2 bar. The Mach number is reduced by $4.7 \%$ as $\mathrm{Re}_{\mathrm{p}}$ decreases from 10000 to 500. For the particle layer lengths examined here, the additional

TABLE I. Average ratio of viscous to pressure forces acting on the particles (force ratio) over the time frame where the shock wave interacts with each particle, the Mach numbers of the transmitted $\left(\mathrm{Ma}_{\mathrm{TS}}\right)$ and the reflected $\left(\mathrm{Ma}_{\mathrm{RS}}\right)$ shock waves, the particle Reynolds number based on the flow state behind the reflected shock wave $\left(\operatorname{Re}_{\mathrm{p}, \mathrm{RS}}\right)$, and the local Mach number of the flow behind the reflected shock wave $\left(\mathrm{Ma}^{*}\right)$.

\begin{tabular}{lccccc}
\hline \hline $\mathrm{Re}_{\mathrm{p}}$ & Force ratio & $\mathrm{Ma}_{\mathrm{TS}}$ & $\mathrm{Ma}_{\mathrm{RS}}$ & $\mathrm{Re}_{\mathrm{p}, \mathrm{RS}}$ & $\mathrm{Ma}^{*}$ \\
\hline 500 & 0.144 & 2.069 & 1.579 & 361 & 0.380 \\
2000 & 0.066 & 2.130 & 1.561 & 1488 & 0.400 \\
5000 & 0.038 & 2.158 & 1.546 & 3811 & 0.417 \\
10000 & 0.026 & 2.171 & 1.534 & 7763 & 0.430 \\
\hline \hline
\end{tabular}


shock-wave attenuation by viscous effects is only moderate, but it might become significant if longer layers are considered.

The position of the reflected shock wave as a function of time is also shown in Fig. 3. The location of the reflected shock wave is identified from the volume-averaged pressure and defined as the location where the pressure is the average of the upstream pressure and the pressure behind the reflected shock. Since the incident flow is supersonic, the reflected shock wave moves slowly in this reference frame. Its strength increases with decreasing Reynolds number. This is expected, since the magnitude of the particle drag increases with reduced Reynolds number. There is a larger resistance for the flow to pass through the particle layer due to the viscous forces, which builds up pressure in the flow. Therefore the reflected shock strength must increase. Note that the strength of the reflected shock increases with time.

The average Mach number of the reflected shock is given in Table I. It is based on the average speed of the reflected shock wave over the time frame where it is located upstream of $x=-0.1 L$. The lower Reynolds numbers have higher reflected shock Mach numbers, and the difference between $\operatorname{Re}_{\mathrm{p}}=10000$ and $\operatorname{Re}_{\mathrm{p}}=500$ is $2.9 \%$.

Table I also shows the particle Reynolds number and the local Mach number of the flow based on the properties behind the reflected shock, assuming a constant reflected shock Mach number. For classification of the late-time flow field in the particle cloud, these two parameters are more appropriate than the Mach number behind the incident shock wave and $\operatorname{Re}_{\mathrm{p}}$ based on $\rho_{\mathrm{IS}}, u_{\mathrm{IS}}$, and $\mu_{\mathrm{IS}}$. The change in Reynolds number is moderate and does not imply any qualitative changes in the flow, based on the characterization in single-particle studies. The change in local Mach number is more important because the flow behind the incident shock is supersonic, while the flow behind the reflected shock is subsonic with a relatively low Mach number.

\section{Flow fields and fluctuations}

Figure 4 shows the normalized streamwise velocity, pressure, and fluctuating kinetic energy at three different time points. The fluctuating kinetic energy is normalized by the inflow kinetic energy. Similarly, Fig. 5 shows the Mach number, particle Reynolds number, and normalized average streamwise particle forces,

$$
F_{\mathrm{p}}=\frac{1}{0.5 N_{\mathrm{p}}(x)\left(\rho u^{2}\right)_{\mathrm{IS}} A_{\mathrm{p}}} \sum_{j=0}^{N_{\mathrm{p}}(x)} \int_{S_{j}}\left(p n_{1}-\sigma_{1 k} n_{k}\right) d S,
$$

where $N_{\mathrm{p}}(x)$ is the number of particles within the control volume centered at $x$, and $S_{j}$ is the surface of particle $j$. Figure 5 also shows the ratio of viscous particle forces to total particle forces.

The fluctuating kinetic energy is the only flow quantity in Figs. 4 and 5 that has a nonmonotone dependence on $\mathrm{Re}_{\mathrm{p}}$. The three highest Reynolds numbers have very similar fluctuating kinetic energies at all times, but at the latest time point $\operatorname{Re}_{\mathrm{p}}=2000$ attains slightly higher fluctuation levels than $\mathrm{Re}_{\mathrm{p}}=5000$ and $\mathrm{Re}_{\mathrm{p}}=10000$ in most of the domain. The level of fluctuating kinetic energy was also considered in inviscid flows by Mehta et al. [22]. Comparison of the current results to the results reported by Mehta et al. [22] indicates that the fluctuations are higher in viscous than inviscid flows, which is a result caused by the no-slip condition at the particle surface and the flow separation. The fluctuation levels are in fact comparable to the levels in the inviscid simulations at $\alpha_{\mathrm{p}}=0.20$ and $\mathrm{Ma}=1.6$ by Mehta et al. [22], i.e. at twice the volume fraction of the current simulations.

The Mach number plots reveal that the flow becomes sonic just upstream of the downstream particle cloud edge. Similar observations have been made in other studies [19,27,31], and this behavior is very similar to that found in Fanno flows. As discussed in Emanuel and Jones [38], the Mach number increases with downstream distance in Fanno flows due to the decreasing pressure. If the duct is sufficiently long, the flow chokes at the downstream exit, just like we observe here. 

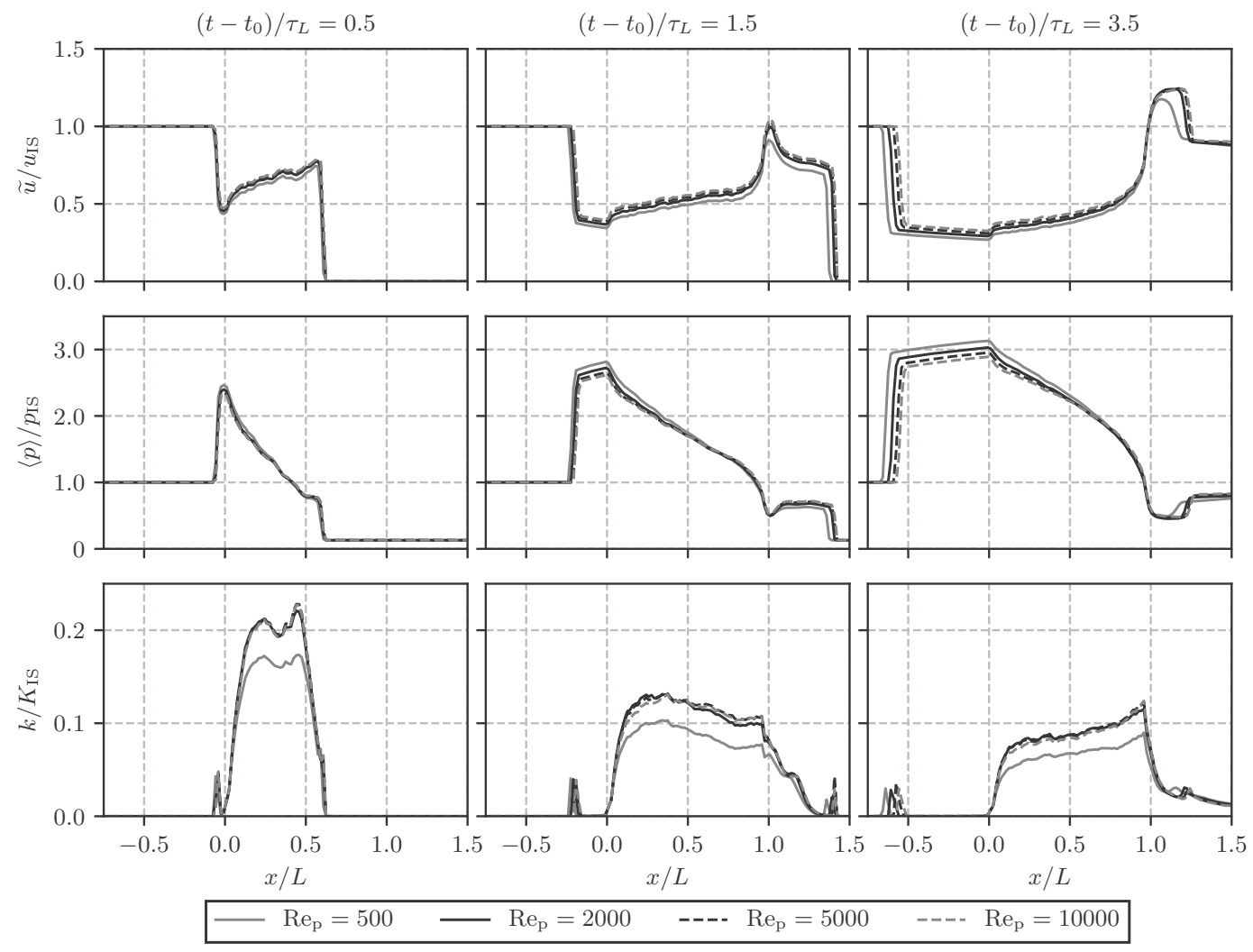

FIG. 4. Streamwise velocity, pressure, and fluctuating kinetic energy at three times for different $\mathrm{Re}_{\mathrm{p}}$. The first column shows a time where the shock wave is still inside the particle layer, the second where it has recently exited the layer, and the final column is close to the end of the simulation.

In Fig. 5 it can be seen that the particle forces increase drastically at the downstream layer edge for the two latest time points shown. The Reynolds number increases slightly as well but is insufficient for explaining the increase in particle forces. Instead, the higher particle forces are due to the increased Mach number.

The ratio of viscous forces and total forces on the particles increases when $\mathrm{Re}_{\mathrm{p}}$ decreases. This trend has also been reported for single-particle studies [46]. It can be seen that the ratio decreases in the region where the Mach number increases. Nagata et al. [35] reported that the pressure drag coefficient increases significantly with Mach number, while the viscous drag coefficient has a more modest increase. The current results agree with this finding, and it explains the reason for the dip in force ratio. The results for $\operatorname{Re}_{\mathrm{p}}=500$, which have local Reynolds numbers just below 250 at $\left(t-t_{0}\right) / \tau_{L}=3.5$, are also in good quantitative agreement with the results of Nagata et al. [35]. Linear interpolation of the ratio of viscous and pressure forces from their simulations at $\operatorname{Re}_{\mathrm{p}}=250$ predicts that the ratio should drop from 0.38 at $\mathrm{Ma}=0.5$ to 0.29 at $\mathrm{Ma}=1.0$, which is quite similar to the drop in the current simulations; cf. Fig. 5.

An important part of the high speed flow through particle clouds is the particle-scale fluctuations. These are commonly neglected in Eulerian-Lagrangian and Eulerian-Eulerian simulations of shockwave particle cloud interactions; see, e.g., Houim and Oran [14], Ling et al. [16], Sugiyama et al. [18], and Theofanous and Chang [19]. The gradient of the streamwise velocity fluctuations is an integral part of the momentum balance around the particle cloud edges. Osnes et al. [31] showed that at the upstream particle cloud edge, the streamwise Reynolds stress gradient is of equal importance 

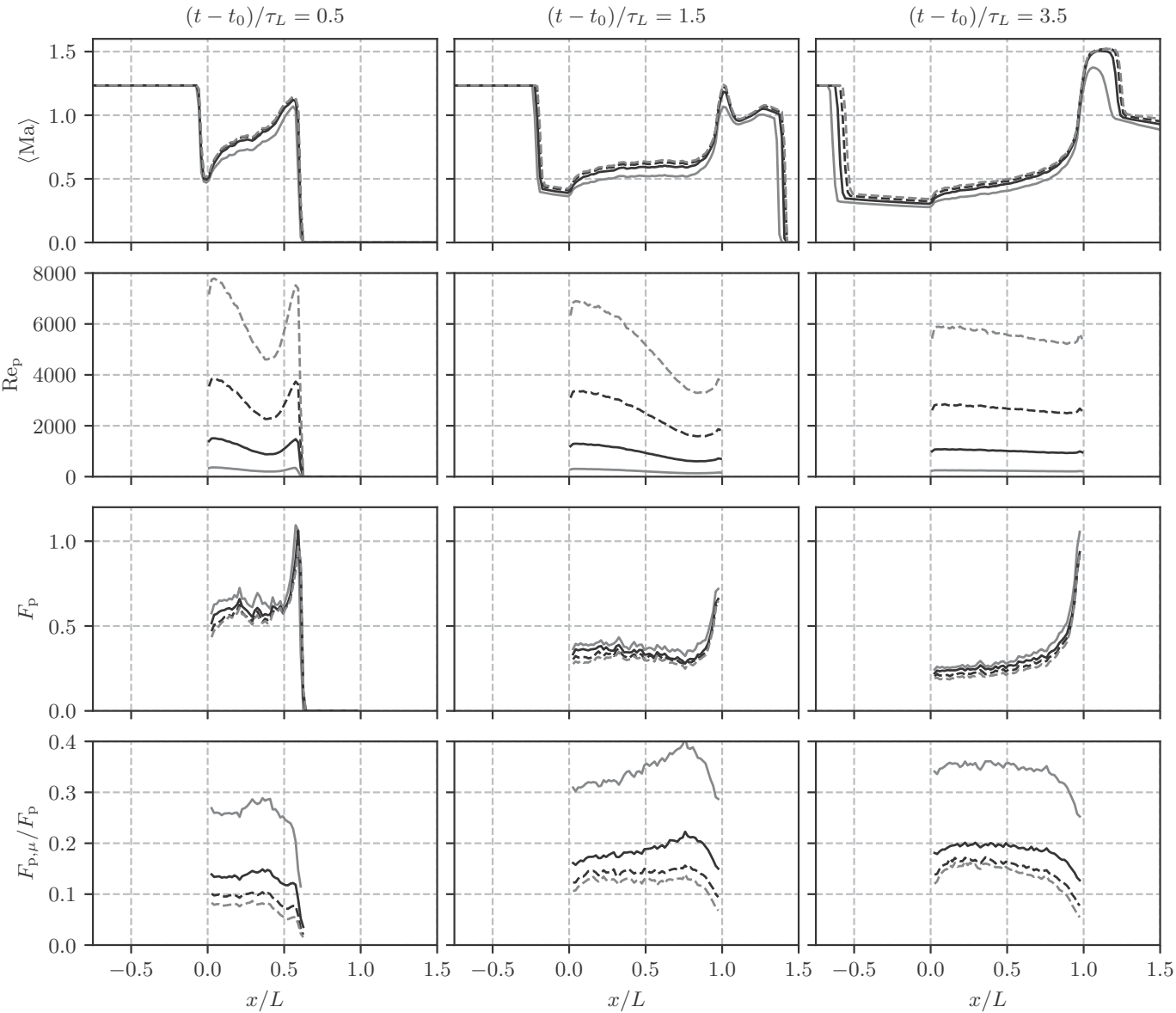

$$
\square \operatorname{Re}_{\mathrm{p}}=500 \quad \longrightarrow \operatorname{Re}_{\mathrm{p}}=2000 \quad---\operatorname{Re}_{\mathrm{p}}=5000 \quad---\operatorname{Re}_{\mathrm{p}}=10000
$$

FIG. 5. Mach number, local particle Reynolds number, average normalized particle forces, and ratio of viscous $\left(F_{\mathrm{p}, \mu}\right)$ and total particle forces at three time points for different $\mathrm{Re}_{\mathrm{p}}$. The first column shows a time point where the shock wave is still inside the particle layer, the second where it has recently exited the layer, and the final column close to the end of the simulation.

as the particle forces after the initial shock-related transient. As can be seen in Fig. 4, the fluctuating kinetic energy has a very sharp gradient, and its magnitude is considerable, especially at early times. Figure 6 shows the ratio of fluctuating kinetic energy to total kinetic energy, integrated over the interior of the particle cloud, as a function of time. It can be seen that the ratio exceeds 0.25 for all Reynolds numbers around $\left(t-t_{0}\right) / \tau_{L}=0.5$, i.e., when the shock wave is slightly less than halfway through the particle cloud. When the shock wave exits the cloud, the ratio decays slowly over time but remains above 0.2 for all $\mathrm{Re}_{\mathrm{p}}$ over the time frame considered here. The trend with $\mathrm{Re}_{\mathrm{p}}$ is not monotone, which is also the case for the fluctuating kinetic energy; cf. Fig. 4.

Regele et al. [25] showed that the pressure field computed in a one-dimensional EulerianLagrangian simulation of shock-wave particle cloud interaction corresponded to the sum of the volume-averaged pressure and the Reynolds stress in their two-dimensional simulations. The overestimation of the pressure in their one-dimensional simulations is therefore the result of neglecting the energy deposited in velocity fluctuations. For the current simulations, the ratio $k / p$ takes values up to 0.25 when the shock wave is inside the particle cloud. It remains non-negligible 


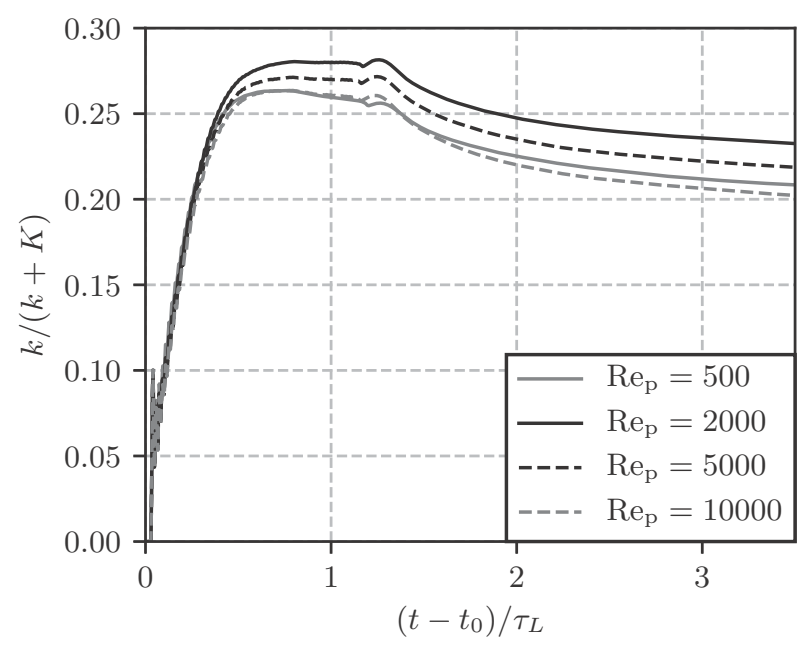

FIG. 6. Ratio of fluctuating to total kinetic energy integrated over the particle layer, as a function of time, for different Reynolds numbers.

even at late times, where the value is between 0.02 and 0.14 within the particle cloud. The ratio is lower for $\mathrm{Re}_{\mathrm{p}}=500$ than the other Reynolds numbers, which are quite similar.

The fluctuation levels at any location within the particle cloud are highly transient when, and for a while after, the shock passes through this location. Figures 7 to 9 show the streamwise velocity fluctuations over time as well as velocity contours at selected time points at streamwise locations $x / L=0.1$ (Fig. 7), $x / L=0.5$ (Fig. 8), and $x / L=0.95$ (Fig. 9). The fluctuation level rises sharply after the shock passes, reaches a peak value after a delay of about $3 \tau_{\mathrm{p}}$, before decaying
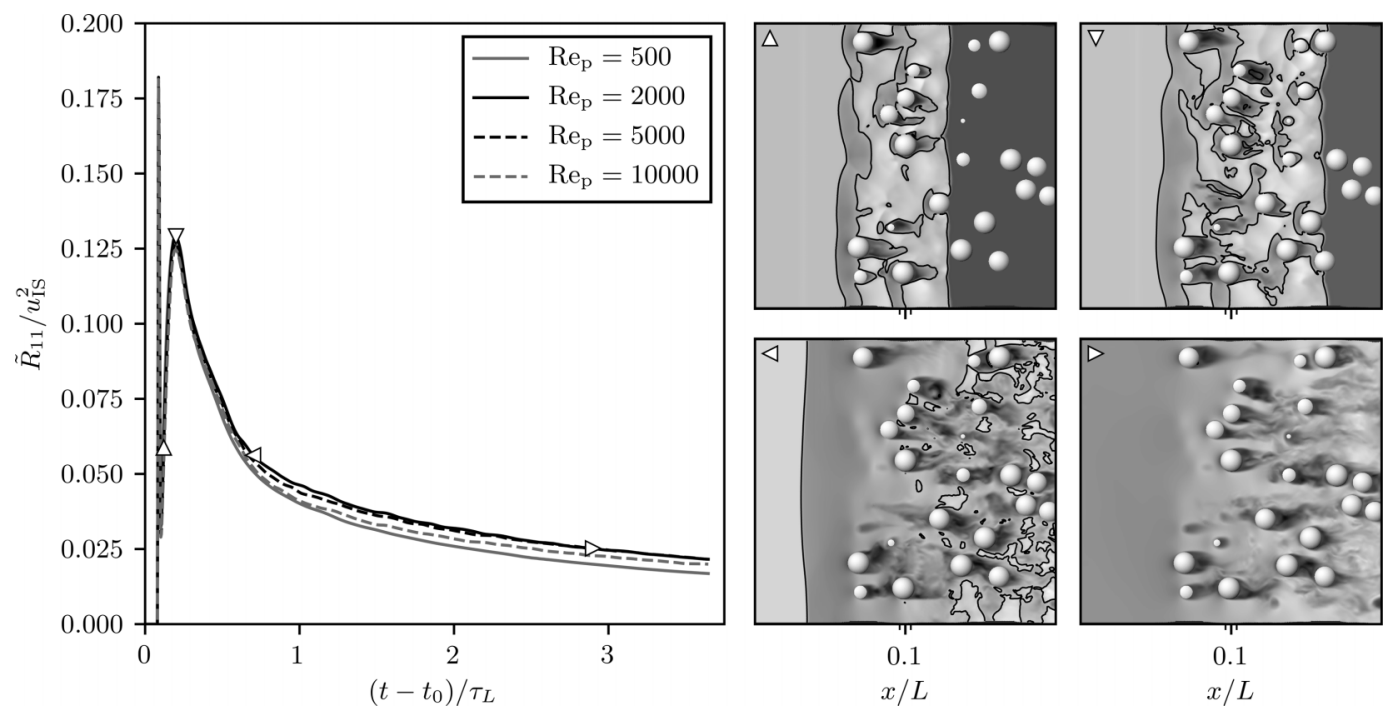

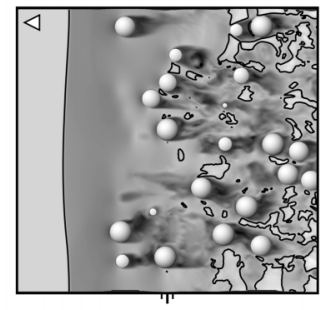

0.1

$x / L$

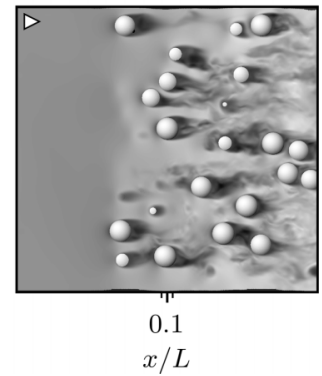

FIG. 7. Streamwise velocity fluctuation correlations over time $x / L=0.1$, and streamwise velocity contours for $\mathrm{Re}_{\mathrm{p}}=2000$ at four time points. Grayscale contour levels between $-0.7 u_{\mathrm{IS}}$ (black) and 1.6 $u_{\mathrm{IS}}$ (white) in $(\triangle),-0.9 u_{\mathrm{IS}}$ (black) and $1.6 u_{\mathrm{IS}}$ (white) in $(\nabla),-0.5 u_{\mathrm{IS}}$ (black) and $1.3 u_{\mathrm{IS}}$ (white) in $(\triangleleft)$, and between $-0.3 u_{\mathrm{IS}}$ (black) and $0.8 u_{\mathrm{IS}}$ (white) in $(\triangleright)$. The solid black lines in the contour plots mark Ma $=1$. The minor ticks denote the streamwise extent of the averaging volume centered at $x / L=0.1$. 

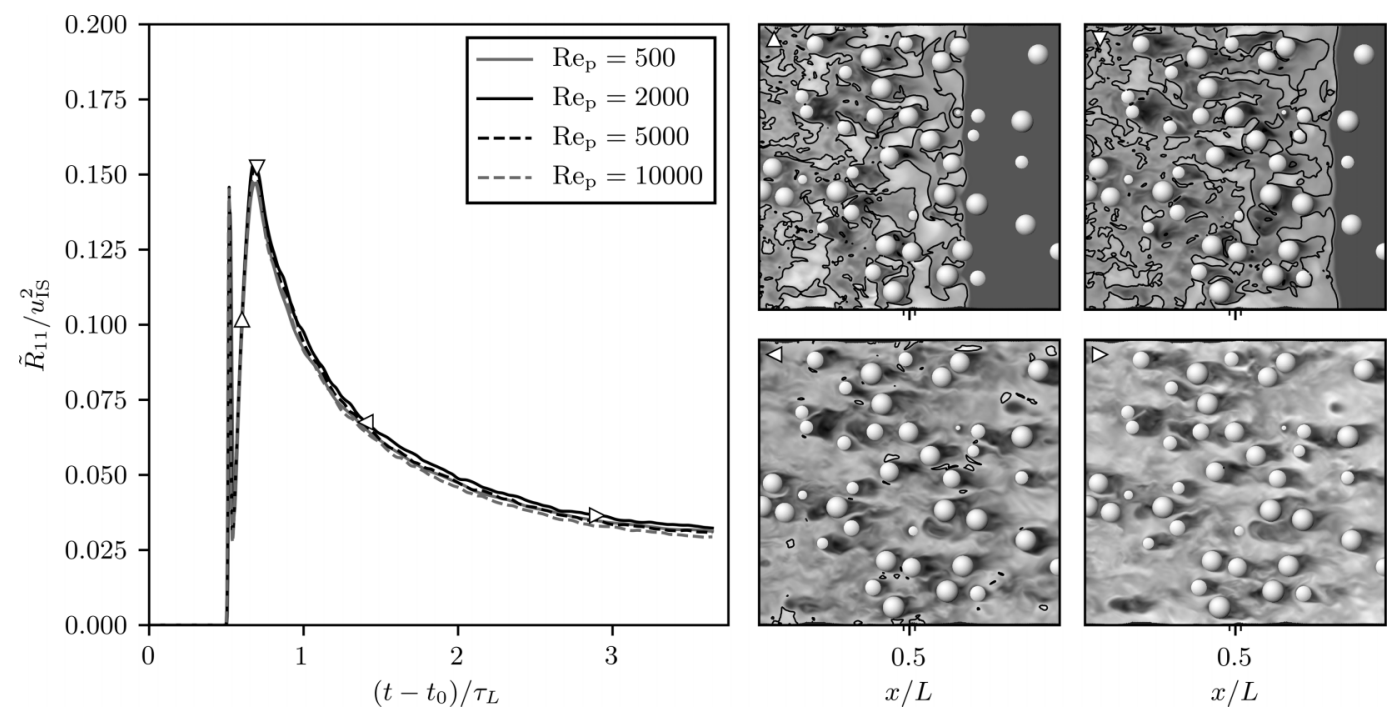

FIG. 8. Streamwise velocity fluctuation correlations over time $x / L=0.5$, and streamwise velocity contours for $\mathrm{Re}_{\mathrm{p}}=2000$ at four time points. Grayscale contour levels between $-0.8 u_{\mathrm{IS}}$ (black) and 1.6u $u_{\mathrm{IS}}($ white) in $(\triangle),-0.8 u_{\mathrm{IS}}$ (black) and $1.8 u_{\mathrm{IS}}$ (white) in $(\nabla),-0.4 u_{\mathrm{IS}}$ (black) and $1.25 u_{\mathrm{IS}}$ (white) in $(\triangleleft)$, and between $-0.35 u_{\mathrm{IS}}$ (black) and $1.85 u_{\mathrm{IS}}$ (white) in $(\triangleright)$. The solid black lines in the contour plots mark Ma $=1$. The minor ticks denote the streamwise extent of the averaging volume centered at $x / L=0.5$.

towards a quasisteady fluctuation level at late times. The peak value coincides with the breakdown of supersonic flow pockets between the particles, as can be seen in the contour plots of the flow state at the time of the peak. In the averaging volume that contains the transition from subsonic to supersonic flow, the flow accelerates both in front of and behind each particle due to the area contraction and expansion. The flow acceleration itself creates large velocity differences within the averaging volume, as well as amplifying the difference between the velocity in the separated flow behind the particle and that around it. Prior to the peak, most of the flow is supersonic, with only small subsonic regions behind the particles. After the peak, the flow becomes predominantly subsonic at locations within the cloud, while it remains supersonic at late times downstream of the cloud as seen in Fig. 9.

Comparison of Figs. 7-9 reveals that the peak fluctuation level is higher in the central region of the cloud than close to the edges. At $x / L=0.95$, the difference between the peak fluctuation level and the later quasisteady value is much smaller, due to the elevated level of the latter. This can be attributed to the strong flow acceleration over the downstream cloud, which in fact becomes stronger over time; cf. Fig. 4.

The very sharp first peak in the velocity fluctuation level occurs when the shock wave passes through the averaging volume. This peak is reduced with downstream distance, consistent with the attenuation of the shock wave as is passes through the cloud. The exact peak value might not be well resolved since it is very sensitive to the sampling time. Overall, the temporal variation of the fluctuation level is similar at all Reynolds numbers, but the levels are highest at $\operatorname{Re}_{\mathrm{p}}=2000$.

Currently, there are very few models for flow field fluctuations that have been developed specifically for shock-wave particle cloud interaction. It is therefore useful to accurately identify flow phenomena that contribute to the fluctuation correlations, so that model development can be based on a fundamental understanding of the flow. In the current work, we consider only the velocity fluctuations. Figure 10 shows the contribution to the Reynolds stress by velocity fluctuations of 

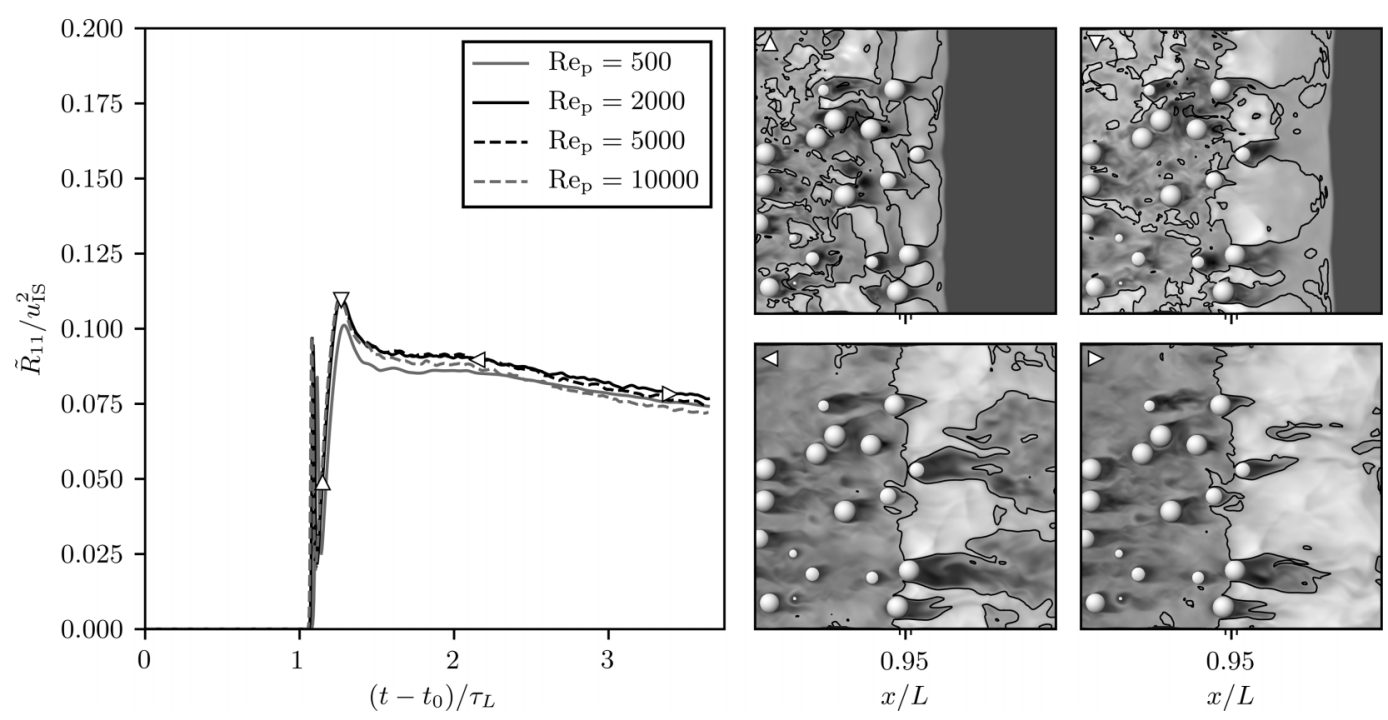

FIG. 9. Streamwise velocity fluctuation correlations over time at $x / L=0.95$, and streamwise velocity contours for $\mathrm{Re}_{\mathrm{p}}=2000$ at four time points. Grayscale contour levels between $-0.6 u_{\mathrm{IS}}$ (black) and $1.5 u_{\mathrm{IS}}$ (white) in $(\triangle),-0.6 u_{\mathrm{IS}}$ (black) and $1.4 u_{\mathrm{IS}}$ (white) in $(\nabla),-0.5 u_{\mathrm{IS}}$ (black) and $1.7 u_{\mathrm{IS}}$ (white) in $(\triangleleft)$, and between $-0.5 u_{\mathrm{IS}}$ (black) and $1.7 u_{\mathrm{IS}}$ (white) in $(\triangleright)$. The solid black lines in the contour plots mark Ma $=1$. The minor ticks denote the streamwise extent of the averaging volume centered at $x / L=0.95$.

different magnitudes at $t=3.75 \tau_{L}$. The figure shows the function $f$, which is defined by

$$
\langle\rho\rangle \tilde{R}_{11}=\int_{-\infty}^{\infty} f\left(u^{\prime \prime}\right) d u^{\prime \prime} .
$$

$f$ is a measure of the importance of velocity fluctuations of a given amplitude with regard to the velocity fluctuation correlations. For $\operatorname{Re}_{\mathrm{p}}=500$, the peak in $f$ is very sharp, and the contribution from negative fluctuations below the peak is negligible. As the Reynolds number increases, the peak decreases. It moves towards more negative fluctuations because the mean flow velocity increases. The peak also broadens, and the contribution from strong negative fluctuations increases. The
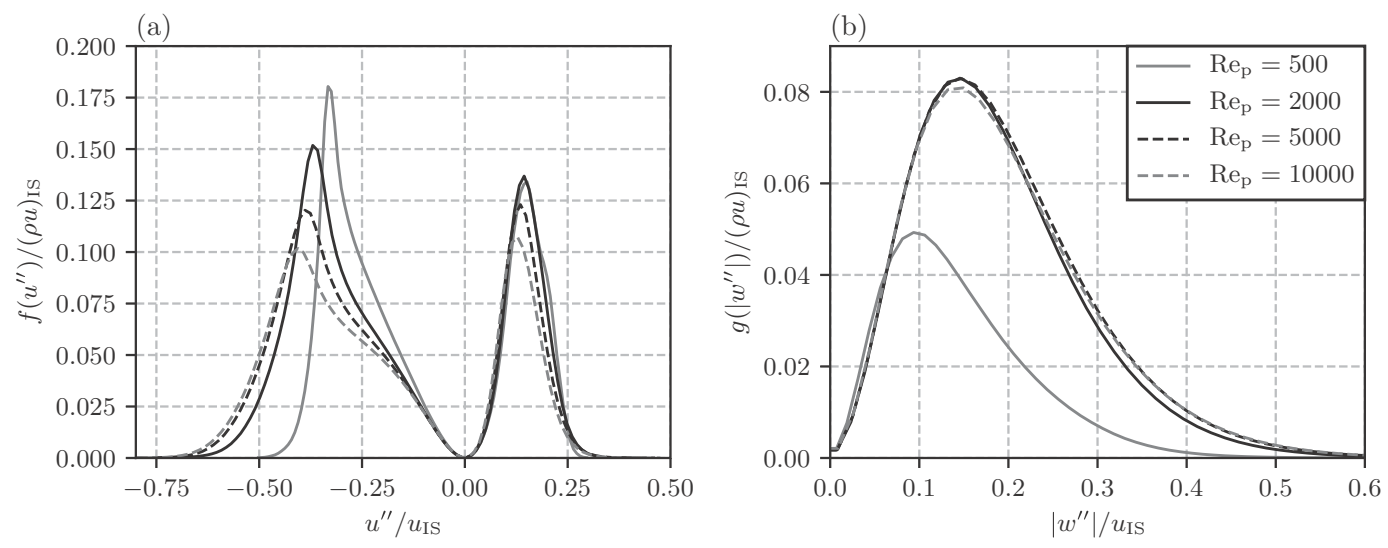

FIG. 10. Contribution to (a) the streamwise Reynolds stress and (b) the spanwise Reynolds stresses by different velocity fluctuations for different Reynolds numbers. Data for $0.25 \leqslant x / L \leqslant 0.75$ at $t=3.75 \tau_{L}$. 

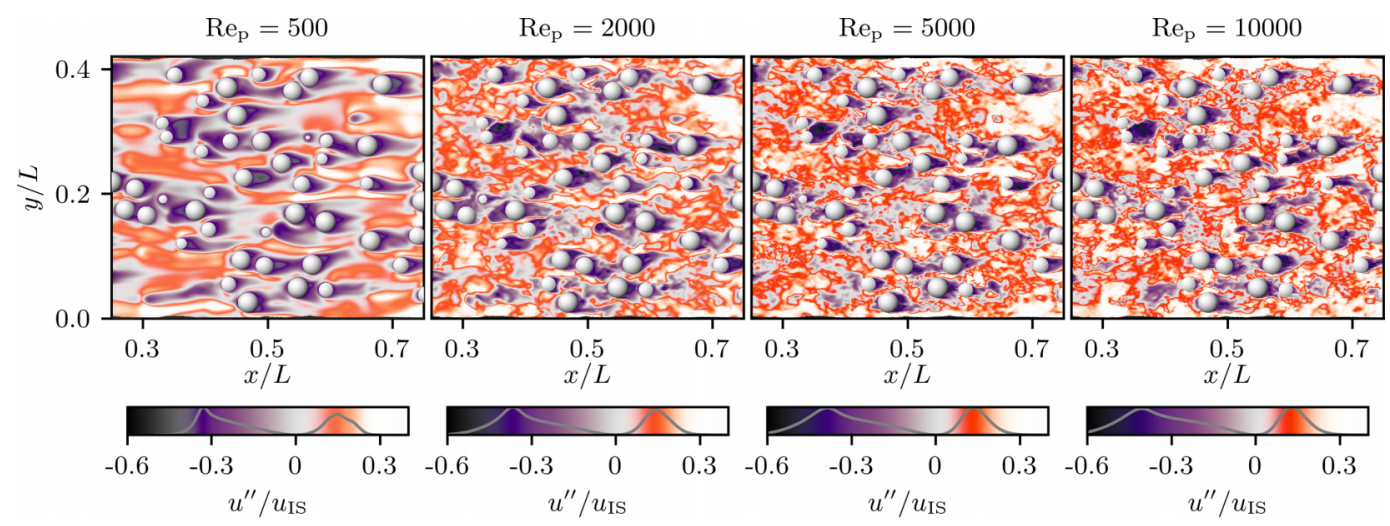

FIG. 11. Contours of $f$ at $t=3.75 \tau_{L}$ for one realization at each $\mathrm{Re}_{\mathrm{p}}$. The color indicates the velocity fluctuation, and $f$ is used to set the color saturation, as indicated by the curve in each colorbar located below the plot. Thus areas with high color saturation have the highest contribution to the Reynolds stress.

total contribution by negative fluctuations increases with Reynolds number, comprising $62.8 \%$, $67.0 \%, 68.7 \%$, and $69.8 \%$ for Reynolds numbers 500, 2000, 5000, and 10000 , respectively. The contribution from positive fluctuations can be seen to have a nonmonotone dependence on Reynolds number over the range studied here. The peak value is very similar for the two lowest Reynolds numbers. When the Reynolds number is increased further, the peak value decreases to $90 \%$ and $78 \%$ of the $\mathrm{Re}_{\mathrm{p}}=2000$ peak for $\mathrm{Re}_{\mathrm{p}}=5000$ and 10000 , respectively. Similarly to the negative fluctuations, the curve broadens, but the effect is small.

A similar plot of $f$ was used in Osnes et al. [31] to argue that the bulk contribution to the Reynolds stress was due to the circulation region behind each particle. Those results were for a flow configuration very similar to the $\mathrm{Re}_{\mathrm{p}}=5000$ case considered here. Figure 11 shows contours of $f$ in space and confirms that the circulation regions are those with the highest contributions to the streamwise Reynolds stress. The flow in the circulation region has an average velocity of zero and therefore differs drastically from the mean flow speed. This implies that the volume of the circulation region is an important parameter for determining the streamwise Reynolds stress magnitude. It is also evident that most of the flow volume contains velocities that are higher than the volume-averaged velocity. This effect arises because the separation regions shift the average velocity away from the velocity of the flow between the particles. At $\operatorname{Re}_{\mathrm{p}} \geqslant 2000$ there are very few areas within the particle cloud that have a velocity equal to the volume-averaged velocity.

Figure 10 also shows the contribution to the spanwise Reynolds stresses by spanwise velocity fluctuations of different magnitudes. The function $g\left(\left|w^{\prime \prime}\right|\right)$ is the analog to $f\left(u^{\prime \prime}\right)$ for spanwise fluctuations and is averaged over the two spanwise velocity components. Additionally, since $g\left(\left|w^{\prime \prime}\right|\right)$ should be symmetric around $w^{\prime \prime}=0$, we plot the contribution as a function of the fluctuation magnitude. The shapes of the curves are quite similar for all Reynolds numbers, but the magnitude is significantly lower for $\operatorname{Re}_{\mathrm{p}}=500$. The location of the peak value is at $\left|w^{\prime \prime}\right| / u_{\mathrm{IS}}=0.09$ for $\operatorname{Re}_{\mathrm{p}}=500$ and around $\left|w^{\prime \prime}\right| / u_{\mathrm{IS}}=0.15$ for the other Reynolds numbers. The trend with $\operatorname{Re}_{\mathrm{p}}$ is nonmonotone, and the highest magnitude is obtained at $\mathrm{Re}_{\mathrm{p}}=2000$. This is similar to the trend for positive streamwise fluctuations, but the reduction at higher $\operatorname{Re}_{\mathrm{p}}$ is less significant for the spanwise fluctuations.

The areas that contribute most to the spanwise Reynolds stress components are shown in Fig. 12. It can be seen that at $\mathrm{Re}_{\mathrm{p}}=500$, the fluctuations are located in large coherent regions. These are significantly larger than a single particle, which indicates that it is the interparticle spacing rather than the particle size that is important for the spanwise Reynolds stress components. The same phenomenon is seen for the higher Reynolds numbers but to a lesser degree. At $\operatorname{Re}_{\mathrm{p}}=10000$, the scale of the coherent regions appears only slightly larger than a single particle. The regions 


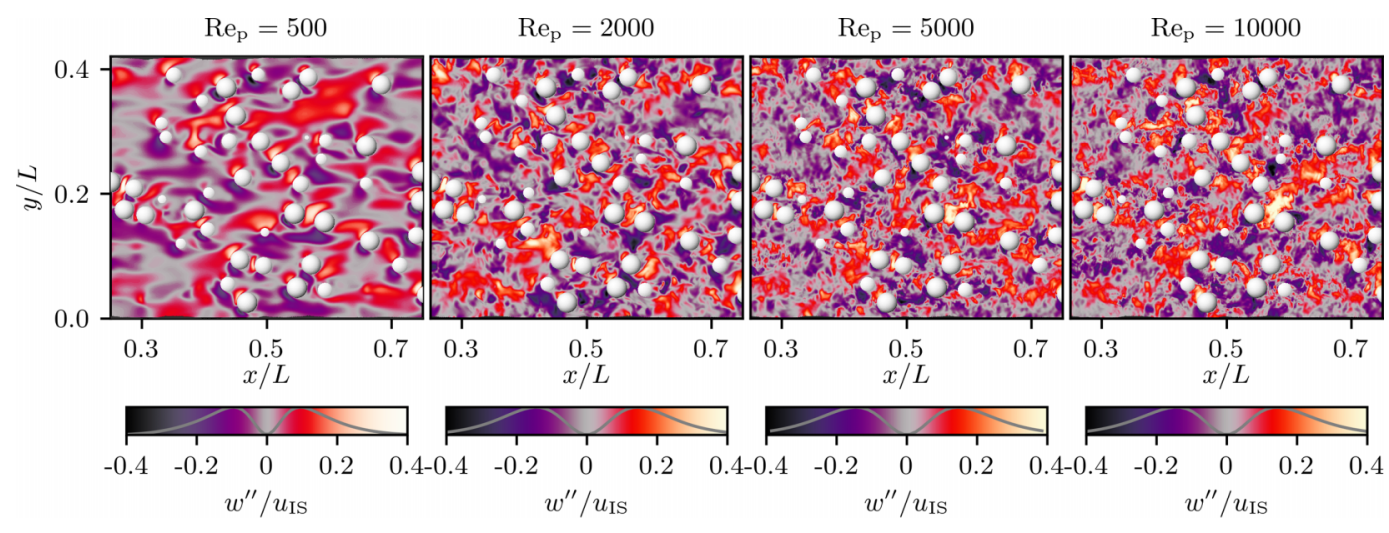

FIG. 12. Contours of $g$ at $t=3.75 \tau_{L}$ for one realization at each $\operatorname{Re}_{\mathrm{p}}$. The color indicates the velocity fluctuation and $g$ is used to set the color saturation, as indicated by the curve in each colorbar located below the plot. Thus areas with high color saturation have the highest contribution to the Reynolds stress.

contributing to the spanwise fluctuations are not the same as those that are important for the streamwise fluctuations. The streamwise fluctuations are primarily particle wakes, whereas the spanwise fluctuations are mostly due to flow deflection around particles and streaming through channels between them. The spanwise fluctuations are therefore located slightly in front and to the side of each particle, as well as in the open spaces within the cloud.

In Figs. 13 and 14, the streamwise and spanwise Reynolds stress contributions are plotted at different streamwise locations within the particle layer. $\mathrm{Re}_{\mathrm{p}}=2000$ and 10000 have very similar trends as $\operatorname{Re}_{\mathrm{p}}=5000$ and are therefore not shown. It can be seen that negative streamwise fluctuations at $\operatorname{Re}_{\mathrm{p}}=500$ have very little variation with streamwise position. On the other hand, positive fluctuations shift towards higher magnitudes as distance increases, and the curve broadens. This is also the case for $\mathrm{Re}_{\mathrm{p}}=5000$, although the shifts are slightly lower. The shift in positive direction is related to the flow acceleration, which is stronger for $\operatorname{Re}_{p}=500$ than for $\operatorname{Re}_{p}=5000$. For $\mathrm{Re}_{\mathrm{p}}=5000$, the shape of the curve for negative fluctuations changes with downstream distance. Smaller magnitudes become more important, presumably due to increasingly turbulent flow.

For spanwise fluctuations, the first half of the cloud features an increase in the contribution at most fluctuation levels, and a slight shift of the peak location towards larger fluctuations. Over
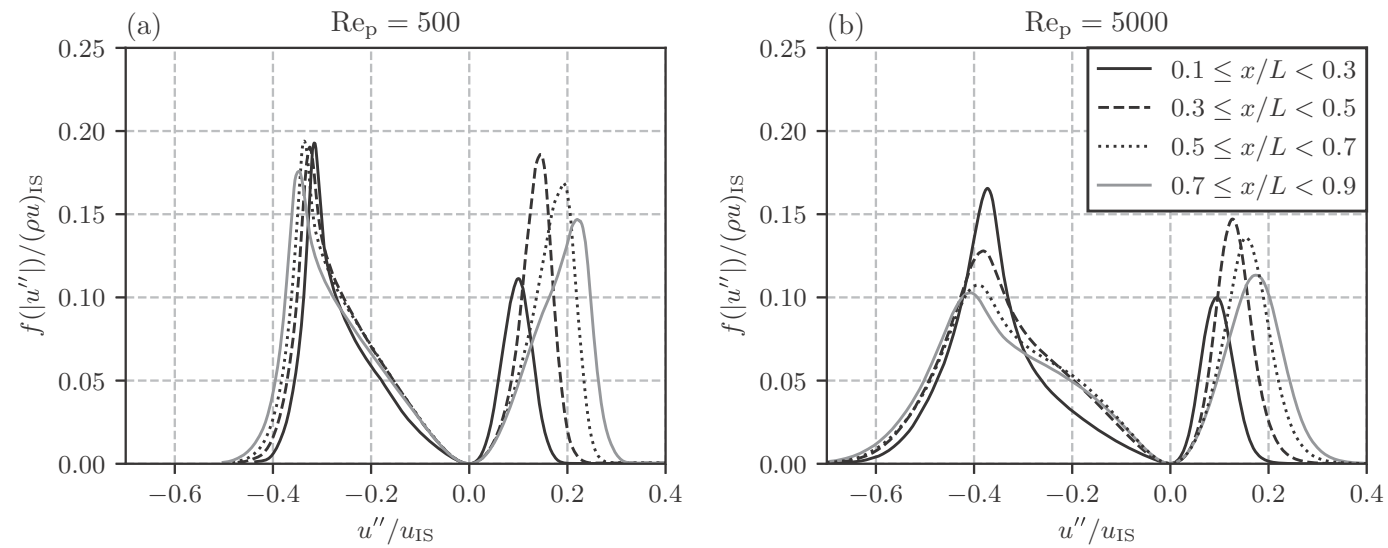

FIG. 13. Contribution to the streamwise Reynolds stress by different velocity fluctuations at $t=3.75 \tau_{L}$ for different streamwise positions. (a) $\operatorname{Re}_{\mathrm{p}}=500$. (b) $\mathrm{Re}_{\mathrm{p}}=5000$. 

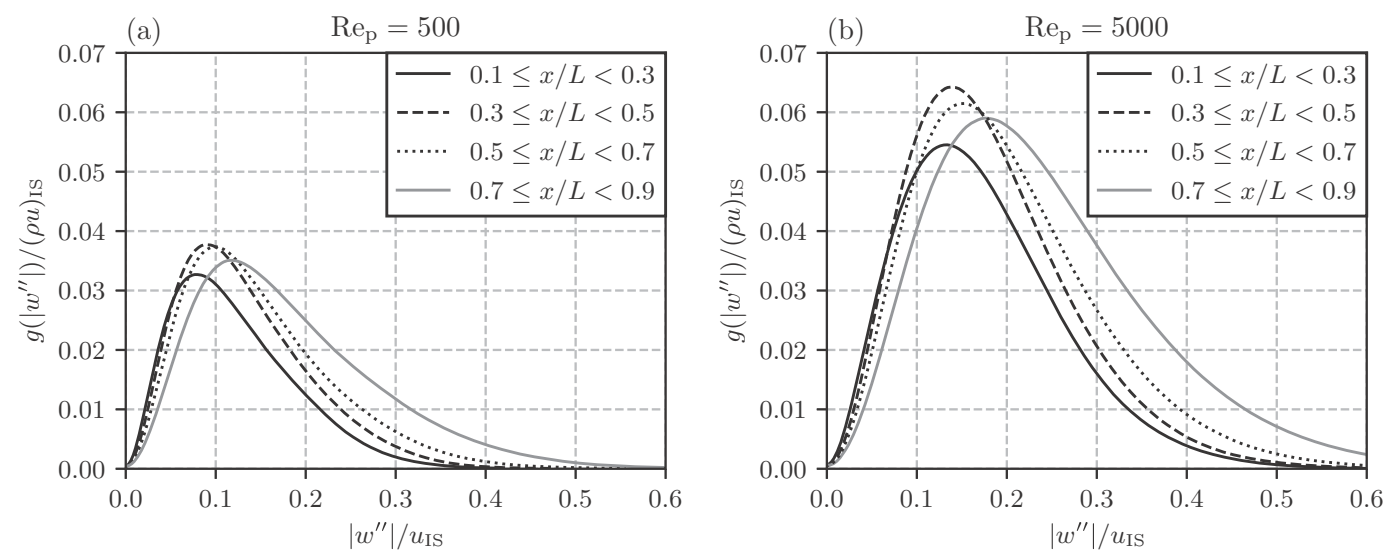

FIG. 14. Contribution to the spanwise Reynolds stress by different velocity fluctuations at $t=3.75 \tau_{L}$ for different streamwise positions. (a) $\operatorname{Re}_{\mathrm{p}}=500$. (b) $\operatorname{Re}_{\mathrm{p}}=5000$.

the downstream half, the peak level diminishes, and its location moves towards larger values. The importance of fluctuations smaller than approximately $0.1\left|w^{\prime \prime}\right| / u_{\mathrm{IS}}$ at $\operatorname{Re}_{\mathrm{p}}=500$ and approximately $0.15\left|w^{\prime \prime}\right| / u_{\mathrm{IS}}$ at $\operatorname{Re}_{\mathrm{p}}=5000$ is reduced, while the contributions by higher values increase.

Osnes et al. [31] found that the streamwise fluctuations in the interior of the particle cloud could be represented by

$$
\left\langle u^{\prime \prime} u^{\prime \prime}\right\rangle=\tilde{u}^{2} \frac{\alpha_{\mathrm{sep}}}{\alpha-\alpha_{\mathrm{sep}}},
$$

where $\alpha_{\text {sep }}$ is an estimate of the average volume of the circulation region behind each particle. This model approximates the flow as two constant states. One is the flow between the particles and the other is the separated flow behind a particle, where the velocity is assumed to be zero. We note that this model does not predict zero velocity fluctuations when $\alpha$ goes to one, nor is it Galilean invariant, and therefore it needs modifications if it is to be used in simplified dispersed flow models. A way to correct this is to replace $\tilde{u}$ by the relative velocity between the particles and the gas, and replace $\alpha_{\text {sep }}$ by $\alpha_{\mathrm{p}} C$ in Eq. (13), where $C$ is a constant or a function of dimensionless flow variables. Figure 15 shows $\alpha_{\text {sep }}$ at the end of each simulation, estimated from the simulation results using Eq. (13). $\alpha_{\text {sep }}$ changes rapidly close to the cloud edges, but over the interior of the cloud it
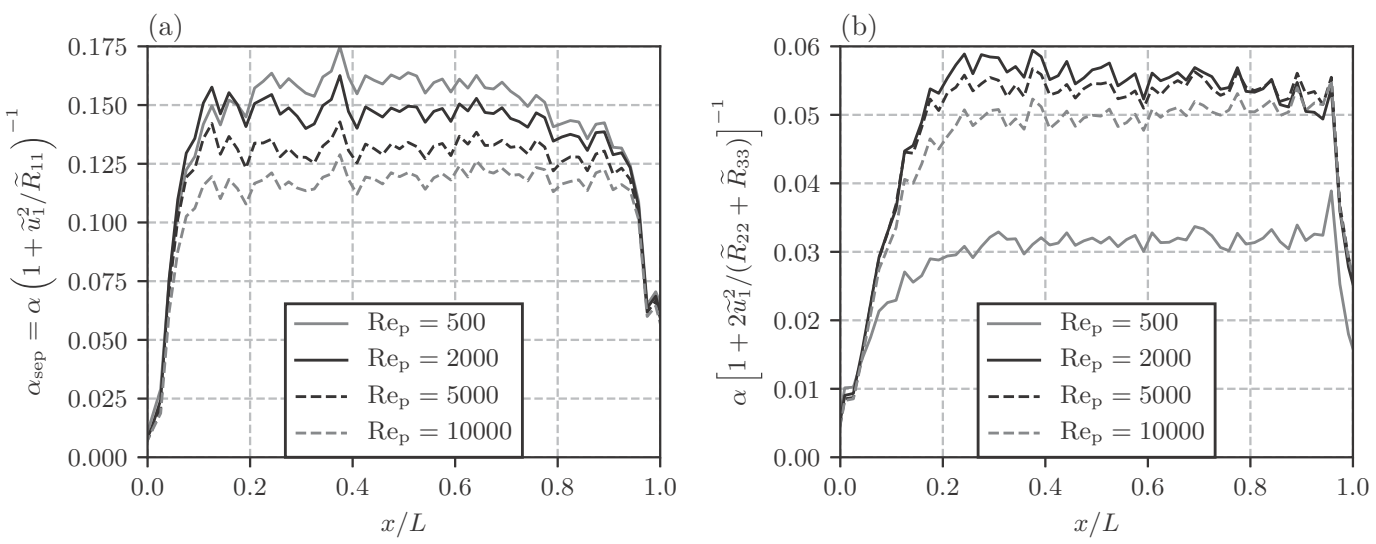

FIG. 15. (a) $\alpha_{\text {sep }}$ and (b) the analogous function for spanwise fluctuations for different $\operatorname{Re}_{\mathrm{p}}$, at $t=3.75 \tau_{L}$. 
has an approximately constant value. $\mathrm{Re}_{\mathrm{p}}=500$ deviates slightly more from a constant value than the higher Reynolds numbers. The trend with $\mathrm{Re}_{\mathrm{p}}$ is monotone over this range, and lower Reynolds numbers have larger values of $\alpha_{\text {sep }}$. The trends found here agree qualitatively with observations from single-sphere studies in the incompressible regime, where the separation length, i.e., the average distance from the particle to the end of the separated flow region, was found to be roughly $30 \%$ shorter at $\operatorname{Re}_{\mathrm{p}}=10000$ than at $\mathrm{Re}_{\mathrm{p}}=3700$ [47]. Indications of a similar trend were also found for compressible flows, where $\mathrm{Re}_{\mathrm{p}}=1000$ at $\mathrm{Ma}=0.8$ and 1.2 had a shorter separation length than $\mathrm{Re}_{\mathrm{p}}=750$ [36].

Figure 15 also shows the analogous function to $\alpha_{\text {sep }}$ for the spanwise fluctuations. It can be seen that this function has little variation over $0.2 \leqslant x / l \leqslant 0.95$. It does not follow from the derivation of Eq. (13) that the spanwise fluctuations should vary this way. The results should be interpreted as confirming that $\left\langle v^{\prime \prime} v^{\prime \prime}\right\rangle$ and $\left\langle w^{\prime \prime} w^{\prime \prime}\right\rangle$ vary proportionally to $\tilde{u}^{2}$ throughout most of the particle layer. We note that for the spanwise fluctuations, there is a longer distance at the start of the layer where the function increases with distance. We suspect that this is because spanwise fluctuations are more related to interparticle distance than particle diameter. If this is the case, it is not surprising that the build-up distance is longer, since the interparticle distance is larger than the particle diameter on average. In contrast to the monotonous behavior of $\alpha_{\text {sep }}$ with $\operatorname{Re}_{\mathrm{p}}$, there is a nonmonotone trend for the spanwise fluctuations.

The simple relationship between the mean flow velocity and the velocity fluctuations is surprisingly robust across different flow conditions. The flow field within the particle cloud features rapidly varying velocity, pressure, and density magnitudes as well as varying gradients. Additionally, the Mach numbers vary from 0.3 to 1 . The insensitivity to these variations is encouraging for development of velocity fluctuation correlation models that can be used as closures for simplified dispersed flow models.

\section{Particle drag}

Accurate description of particle forces is of key importance in dispersed flow simulations. In dense particle suspensions, the local particle configuration can lead to large variations in the directions and magnitudes of the particle forces. The maximal drag for each particle occurs during its interaction with the shock wave. The behavior of maximal drag forces has been examined in inviscid simulations by Mehta et al. [23,24]. We compare the peak streamwise drag forces in the current simulations to their inviscid simulations in Fig. 16. The figure shows the trend in average peak streamwise drag forces with downstream distance and the distribution of peak streamwise drag forces. Note that for the results shown in Fig. 16, the drag forces are presented as a drag coefficient using $\rho_{\mathrm{IS}}$ and $u_{\mathrm{IS}}$, rather than the local values of density and velocity. In Figs. 16-19, subscripts $x$ and $y$ are used to indicate streamwise or spanwise drag coefficients, respectively. The shock-wave Mach number is 2.6 in the current simulations and 3 in the inviscid simulations. We thus expect slightly higher drag coefficients in our simulations. The lower Reynolds number simulations show higher peak drag forces, and this is to be expected because the contribution from viscous forces increases. It can also be seen that the slope is steeper for the lower Reynolds numbers. This is due to the stronger shock-wave attenuation occurring for lower Reynolds numbers. The distribution of the deviation of peak drag forces from the linear trend with $x$ is also shown in Fig. 16. The results are in excellent agreement with the inviscid simulations. It can also be seen that the distribution has only an inconsequential dependence on Reynolds number. This means that the peak drag variation is controlled by processes that are almost unaffected by viscosity, such as shock-wave focusing and diffraction. After the initial shock-induced transient, the importance of viscosity increases. It is therefore of interest to investigate how the distributions of instantaneous and time-averaged drag coefficients depend on Reynolds number. Figure 17 shows the distribution of the drag coefficients averaged over $1.875 \leqslant t / \tau_{L} \leqslant 3.75$. Starting at $t=1.875 \tau_{L}$ ensures that any shock-related transients have decayed. The linear trend with $x$ has been subtracted from the drag coefficients, and they are also normalized with that function. For the spanwise distributions, we 

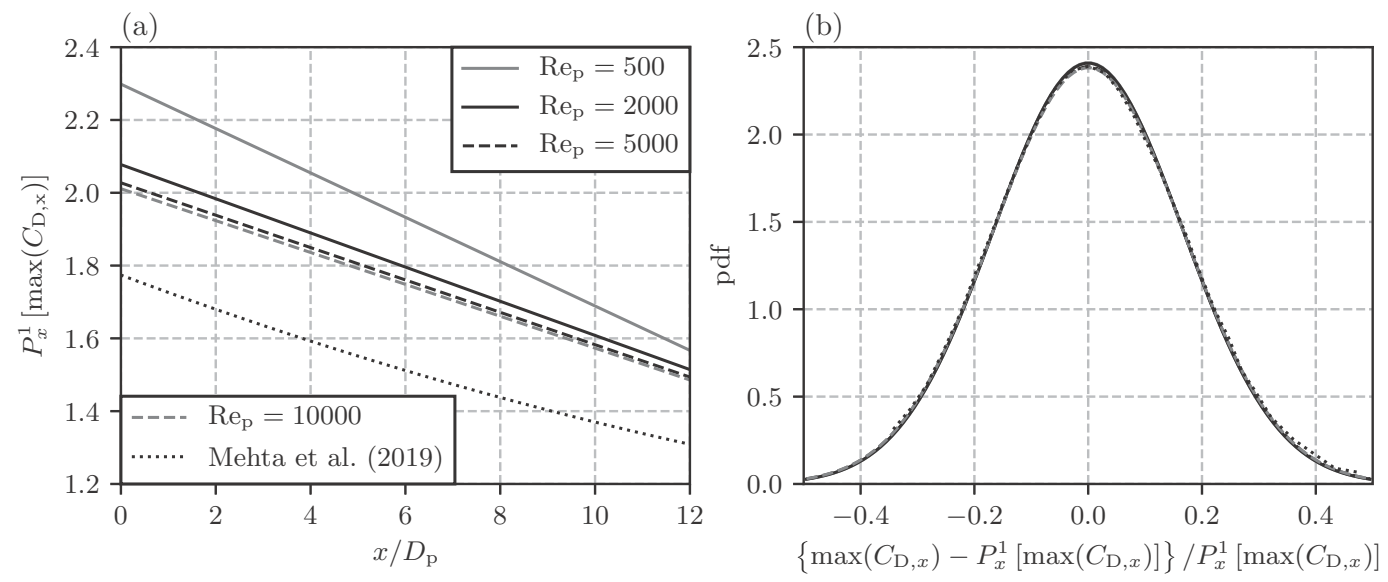

FIG. 16. (a) Least-squares first-order polynomial fit of peak streamwise drag coefficients, denoted $P_{x}^{1}\left[\max \left(C_{\mathrm{D}, x}\right)\right]$, as a function of $x$ for different Reynolds numbers. (b) Best normal distribution fit to the deviation of the peak drag coefficients from $P_{x}^{1}\left[\max \left(C_{\mathrm{D}, x}\right)\right]$. The plots also show the results obtained in inviscid $\mathrm{Ma}=3$ simulations by Mehta et al. [24]. In order to compare against those results, only particles between $0 \leqslant x / D_{\mathrm{p}} \leqslant 12.5$ have been included for the simulations in this work. Note that for the results shown, drag coefficients are based on $\rho_{\mathrm{IS}}$ and $u_{\mathrm{IS}}$.

have normalized the drag coefficients based on the linear trend of the streamwise drag coefficients. The distributions are very similar at all Reynolds numbers, but there is an indication that the distribution is more narrow around $\mathrm{Re}_{\mathrm{p}}=5000$. Since the current distributions are based on a single data point per particle, the relatively small differences between the Reynolds numbers may not be physically meaningful. The distributions are slightly skewed towards positive values. The spanwise distributions are also quite similar for all $\mathrm{Re}_{\mathrm{p}}$. The lowest Reynolds number has the most narrow distribution, while the distributions for higher $\mathrm{Re}_{\mathrm{p}}$ are almost equal.

Figure 18 shows the distribution of the deviation of the instantaneous drag coefficients from a linear trend in time. For each particle, the linear trend in time is also used for normalization. Since the trend in time has been subtracted, these distributions reflect the magnitude and frequency of
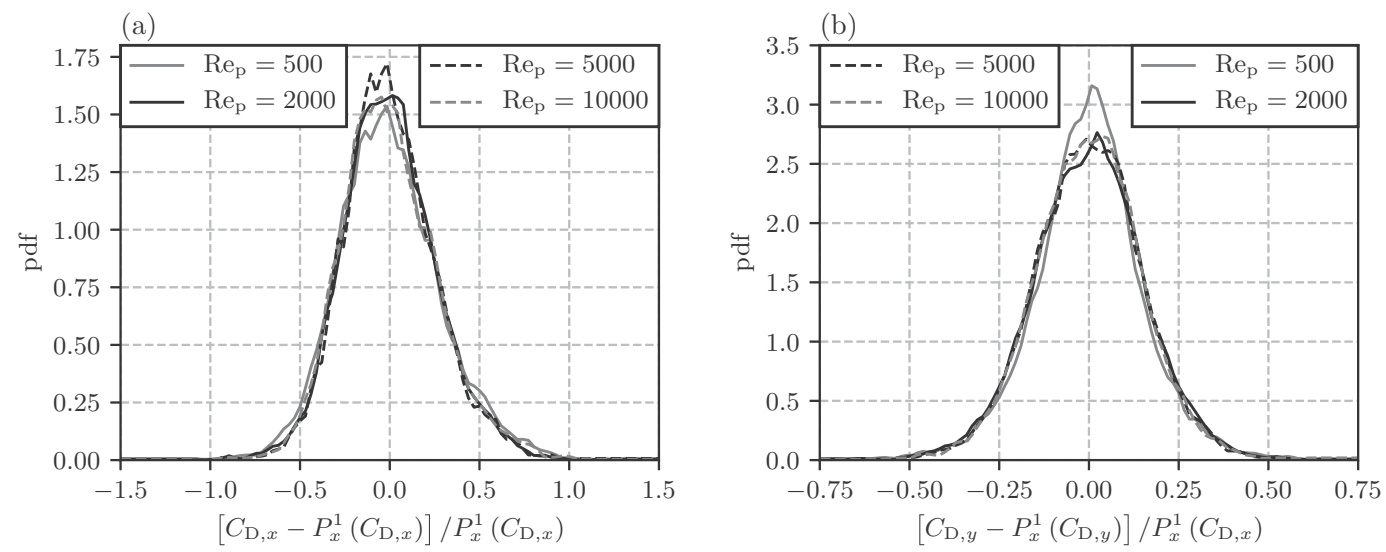

FIG. 17. Distribution of the time-averaged drag coefficients for $t>1.875 \tau_{L}$. (a) Streamwise drag coefficient. (b) Spanwise drag coefficient. The linear trend with $x$ has been subtracted. Note the different scale in the plots. 

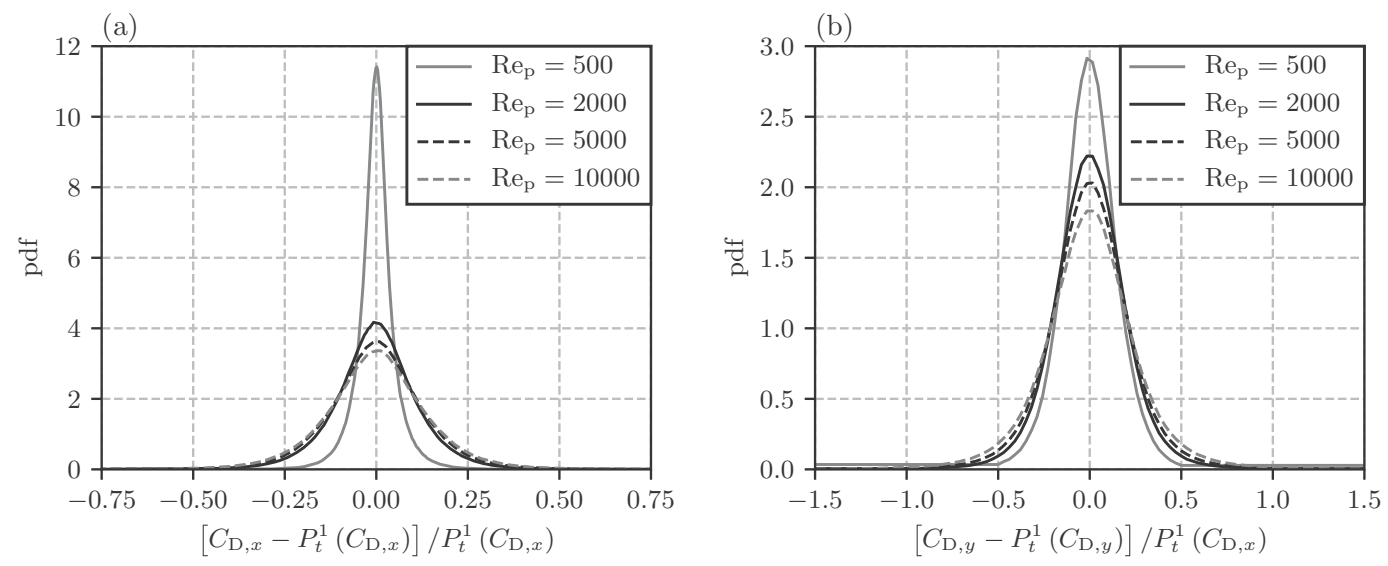

FIG. 18. Distribution of instantaneous drag coefficients for $t>1.875 \tau_{L}$. (a) Streamwise drag coefficient. (b) Spanwise drag coefficient. The linear trend with $t$ for each particle, denoted $P_{t}^{1}\left(C_{\mathrm{D}}\right)$, has been subtracted.

temporal oscillations. The trend with $\mathrm{Re}_{\mathrm{p}}$ is similar for both the streamwise and spanwise drag coefficients, where higher $\mathrm{Re}_{\mathrm{p}}$ result in wider distributions. Note that the spanwise drag coefficient distribution is about twice as wide as that of the streamwise drag coefficient. The trends are more pronounced for the distribution of instantaneous than mean drag coefficients.

Both mean flow and instantaneous drag coefficient distributions have a dependency on particle Reynolds number. There are separate implications for drag modeling from each of these distributions. The distributions of time-averaged drag coefficients imply that drag models should incorporate terms that depend on the local particle configuration. These models can be based on the relative position of nearby particles or on the particle density. The PIEP model developed in Akiki et al. [48] is an example of the former model type. In the latter model type, the distributions shown in Fig. 17 can be used directly by imposing random fluctuations drawn from these distributions. The results here also imply that the importance of including such distributions is equally important at all Reynolds numbers considered here, since the distributions are very similar.

The distribution of instantaneous drag coefficients is a result of temporal flow fluctuations, and these have strong Reynolds number dependencies. It is likely that a part of these distributions is related to the shedding of vortices from both the particle itself and the nearby particles. The variation imposed by vortex shedding from nearby particles could be modeled based on the local particle configuration, although this is likely quite challenging. In addition to the trends with Reynolds number observed here, the distributions are likely dependent on the particle volume fraction. Investigating this effect is a topic for future studies.

Figure 19 shows the streamwise drag coefficient at $t=3.75 \tau_{L}$. The figure also shows the drag coefficients where a modified flow velocity, defined as

$$
u_{\mathrm{free}}=\tilde{u} \frac{\alpha}{\alpha-\alpha_{\mathrm{sep}}},
$$

is used to calculate the drag coefficient. The modified velocity was suggested in Osnes et al. [31] and attempts to correct for the contributions of the circulation regions behind the particles, which shift the average velocity away from the "free" velocity within the particle layer. Also shown in the figure are the single-particle drag coefficients obtained by Nagata et al. [35,36] and the drag correlation by Clift and Gauvin [49]. For reference, the average Mach numbers within the particle cloud are in the range 0.3-0.7; cf. Fig. 5. Here the lower Mach number is for $\operatorname{Re}_{\mathrm{p}}=500$, and the highest Mach number is for $\mathrm{Re}_{\mathrm{p}}=10000$, where the velocity correction has also been used for the Mach number. Even with the correction, which reduces the drag coefficients, the current simulations result in significantly higher drag coefficients than predicted by models based on single-particle results. 


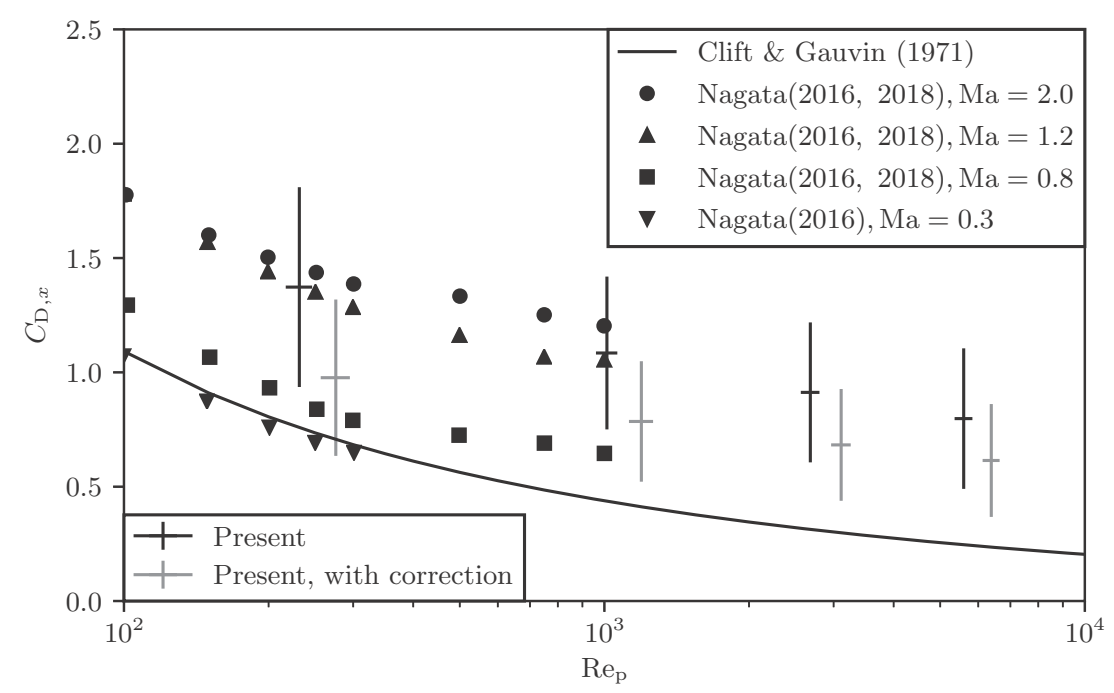

FIG. 19. Average drag coefficient at $t=3.75 \tau_{L}$ for various $\mathrm{Re}_{\mathrm{p}}$, based on local (volume-averaged) flow properties. The standard deviation in $\mathrm{Re}_{\mathrm{p}}$ and $C_{\mathrm{D}, x}$ are indicated by the horizontal and vertical error bars, respectively. The results of Nagata et al. [35,36] as well as the drag correlation of Clift and Gauvin [49] are also shown.

The single-particle simulation results of Nagata et al. [35,36] cover only the lower two Reynolds numbers considered here. However, if the trend from these single-particle simulation results can be extrapolated, it seems that the deviation is larger for low-particle Reynolds numbers than high.

Other studies have found that confinement has a strong effect on the drag on single particles [50-53]. For $\mathrm{Re}_{\mathrm{p}}$ up to about 300, Akutsu [51] found that a $30 \%$ area blockage increased the drag coefficient by more than a factor of two. At $\operatorname{Re}_{\mathrm{p}}$ above $3 \times 10^{4}$, Achenbach [50] found a $10 \%$ increase for $50 \%$ blockage. We expect that a similar effect occurs due to the presence of nearby particles. If a correction for this is combined with the corrections due to Mach number and separation volume, it is likely that the average drag coefficients will approach the values seen in isolated particle studies.

\section{CONCLUDING REMARKS}

This study has investigated the propagation of a Mach 2.6 shock wave through particle clouds with particle volume fraction 0.1. Particle resolved large-eddy simulations were utilized, and the particle Reynolds number, based on the incident shock state, was varied between 500 and 10000 .

The results show that the shock-wave attenuation increases with decreasing particle Reynolds number, but the effect is small over this Reynolds number range. The strength of the reflected shock wave increases with decreasing Reynolds number. This results in considerable changes in the flow quantities upstream of and inside the particle layer, i.e., higher pressures, lower velocities, and lower Mach numbers with reduced particle Reynolds numbers.

We examined the development of fluctuations from the volume-averaged flow quantities within the particle layer. The velocity fluctuation intensity has a nonmonotone dependency on particle Reynolds number. The most intense velocity fluctuations were found at $\mathrm{Re}_{\mathrm{p}}=2000$. Streamwise velocity fluctuations are located in the particle wakes and, most importantly, in the circulation region behind each particle. Spanwise fluctuations are located in the interparticle regions and consist of coherent regions that extend further than the size of single particles. As the Reynolds number increases, the sizes of these regions decrease. We find an approximate proportionality between the mean flow velocity and the flow fluctuations at late times. This relationship holds for 
both the streamwise and the spanwise fluctuations. Interestingly, the proportionality factor varies monotonously with $\mathrm{Re}_{\mathrm{p}}$ for the streamwise fluctuations but not for the spanwise fluctuations.

The distributions of particle forces were examined. Maximal drag forces and their spatial variation agree well with the inviscid simulations of Mehta et al. [23]. In the late-time flow field, we find that the variation of time-averaged particle forces is weakly affected by Reynolds number, while the temporal variation has a strong Reynolds number dependence. These distributions provide a basis for development of particle force variation models that can be used in simplified dispersed flow models.

The particle drag coefficients within the particle cloud were found to be higher than isolated particle drag coefficients on average, and the largest deviation occurred for the lowest Reynolds number. We demonstrated that utilizing a correction to the mean flow velocity yields drag coefficients closer to the single-particle results. A combination of this correction with drag correlations that incorporate Mach number and flow blockage effects can possibly reproduce the observed average drag coefficients in shock-wave particle cloud interactions.

[1] E. J. Chang and K. Kailasanath, Shock wave interactions with particles and liquid fuel droplets, Shock Waves 12, 333 (2003).

[2] S. L. Davis, T. B. Dittmann, G. B. Jacobs, and W. S. Don, Dispersion of a cloud of particles by a moving shock: Effects of the shape, angle of rotation, and aspect ratio, J. Appl. Mech. Tech. Phys. 54, 900 (2013).

[3] Z. Ren, B. Wang, G. Xiang, D. Zhao, and L. Zheng, Supersonic spray combustion subject to scramjets: Progress and challenges, Prog. Aerosp. Sci. 105, 40 (2018).

[4] F. Zhang, D. L. Frost, P. A. Thibault, and S. B. Murray, Explosive dispersal of solid particles, Shock Waves 10, 431 (2001).

[5] A. Chaudhuri, A. Hadjadj, O. Sadot, and G. Ben-Dor, Numerical study of shock-wave mitigation through matrices of solid obstacles, Shock Waves 23, 91 (2013).

[6] A. M. Milne, E. Floyd, A. W. Longbottom, and P. Taylor, Dynamic fragmentation of powders in spherical geometry, Shock Waves 24, 501 (2014).

[7] M. Sichel, C. W. Kauffman, and Y. C. Li, Transition from deflagration to detonation in layered dust explosions, Process Saf. Prog. 14, 257 (1995).

[8] K. Chojnicki, A. B. Clarke, and J. C. Phillips, A shock-tube investigation of the dynamics of gas-particle mixtures: Implications for explosive volcanic eruptions, Geophys. Res. Lett. 33, L15309 (2006).

[9] T. Inoue, R. Yamazaki, and S.-I. Inutsuka, Turbulence and magnetic field amplification in supernova remnants: Interactions between a strong shock wave and multiphase interstellar medium, Astrophys. J. 695, 825 (2009).

[10] D. W. Silvia, B. D. Smith, and J. M. Shull, Numerical simulations of supernova dust destruction. II. Metal-enriched ejecta knots, Astrophys. J. 748, 12 (2012).

[11] E. P. DeMauro, J. L. Wagner, S. J. Beresh, and P. A. Farias, Unsteady drag following shock wave impingement on a dense particle curtain measured using pulse-burst PIV, Phys. Rev. Fluids 2, 064301 (2017).

[12] T. G. Theofanous, V. Mitkin, and C.-H. Chang, The dynamics of dense particle clouds subjected to shock waves. Part 1. Experiments and scaling laws, J. Fluid Mech. 792, 658 (2016).

[13] J. L. Wagner, S. J. Beresh, S. P. Kearney, W. M. Trott, J. N. Castaneda, B. O. Pruett, and M. R. Baer, A multiphase shock tube for shock wave interactions with dense particle fields, Exp. Fluids 52, 1507 (2012).

[14] R. W. Houim and E. S. Oran, A multiphase model for compressible granular-gaseous flows: Formulation and initial tests, J. Fluid Mech. 789, 166 (2016).

[15] G. B. Jacobs, W. S. Don, and T. Dittmann, High-order resolution Eulerian-Lagrangian simulations of particle dispersion in the accelerated flow behind a moving shock, Theor. Comput. Fluid Dyn. 26, 37 (2012). 
[16] Y. Ling, J. L. Wagner, S. J. Beresh, S. P. Kearney, and S. Balachandar, Interaction of a planar shock wave with a dense particle curtain: Modeling and experiments, Phys. Fluids 24, 113301 (2012).

[17] G. S. Shallcross and J. Capecelatro, A parametric study of particle-laden shock tubes using an EulerianLagrangian framework, in Proceedings of AIAA Aerospace Sciences Meeting (American Institute of Aeronautics and Astronautics, Inc., Reston, VA, 2018), p. 2080.

[18] Y. Sugiyama, H. Ando, K. Shimura, and A. Matsuo, Numerical investigation of the interaction between a shock wave and a particle cloud curtain using a CFD-DEM model, Shock Waves 29, 499 (2019).

[19] T. G. Theofanous and C.-H. Chang, The dynamics of dense particle clouds subjected to shock waves. Part 2. Modeling/numerical issues and the way forward, Int. J. Multiph. Flow 89, 177 (2017).

[20] M. G. Omang and J. K. Trulsen, Multi-phase shock simulations with smoothed particle hydrodynamics (SPH), Shock Waves 24, 521 (2014).

[21] D. Lhuillier, C.-H. Chang, and T. G. Theofanous, On the quest for a hyperbolic effective-field model of disperse flows, J. Fluid Mech. 731, 184 (2013).

[22] Y. Mehta, T. L. Jackson, and S. Balachandar, Pseudo-turbulence in inviscid simulations of shock interacting with a bed of randomly distributed particles, Shock Waves 30, 49 (2020).

[23] Y. Mehta, C. Neal, K. Salari, T. L. Jackson, S. Balachandar, and S. Thakur, Propagation of a strong shock over a random bed of spherical particles, J. Fluid Mech. 839, 157 (2018).

[24] Y. Mehta, K. Salari, T. L. Jackson, and S. Balachandar, Effect of Mach number and volume fraction in air-shock interacting with a bed of randomly distributed spherical particles, Phys. Rev. Fluids 4, 014303 (2019).

[25] J. D. Regele, J. Rabinovitch, T. Colonius, and G. Blanquart, Unsteady effects in dense, high speed, particle laden flows, Int. J. Multiph. Flow 61, 1 (2014).

[26] O. Sen, N. J. Gaul, S. Davis, K. K. Choi, G. Jacobs, and H. S. Udaykumar, Role of pseudo-turbulent stresses in shocked particle clouds and construction of surrogate models for closure, Shock Waves $\mathbf{2 8}$, 579 (2018).

[27] T. G. Theofanous, V. Mitkin, and C.-H. Chang, Shock dispersal of dilute particle clouds, J. Fluid Mech. 841, 732 (2018).

[28] P. Das, O. Sen, G. Jacobs, and H. S. Udaykumar, A sharp interface Cartesian grid method for viscous simulation of shocked particle-laden flows, Int. J. Comput. Fluid D 31, 269 (2017).

[29] Z. Hosseinzadeh-Nik, S. Subramaniam, and J. D. Regele, Investigation and quantification of flow unsteadiness in shock-particle cloud interaction, Int. J. Multiph. Flow 101, 186 (2018).

[30] H. Naiman and D. D. Knight, The effect of porosity on shock interaction with a rigid, porous barrier, Shock Waves 16, 321 (2007).

[31] A. N. Osnes, M. Vartdal, M. G. Omang, and B. A. P. Reif, Computational analysis of shock-induced flow through stationary particle clouds, Int. J. Multiph. Flow 114, 268 (2019).

[32] M. Vartdal and A. N. Osnes, Using particle-resolved LES to improve Eulerian-Lagrangian modeling of shock wave particle cloud interaction, in Proceedings of the Summer Program 2018 (Center for Turbulence Research, Stanford, CA, 2018), pp. 25-34.

[33] S. S. Tiwari, E. Pal, S. Bale, N. Minocha, A. W. Patwardhan, K. Nandakumar, and J. B. Joshi, Flow past a single stationary sphere, 2 . Regime mapping and effect of external disturbances, Powder Technol., doi: 10.1016/j.powtec.2019.04.032 (2019).

[34] H. Sakamoto and H. Haniu, A study on vortex shedding from spheres in a uniform flow, J. Fluids Eng. 112, 386 (1990).

[35] T. Nagata, T. Nonomura, S. Takahashi, Y. Mizuno, and K. Fukuda, Investigation on subsonic to supersonic flow around a sphere at low Reynolds number of between 50 and 300 by direct numerical simulation, Phys. Fluids 28, 056101 (2016).

[36] T. Nagata, T. Nonomura, S. Takahashi, Y. Mizuno, and K. Fukuda, Direct numerical simulation of flow past a sphere at a Reynolds number between 500 and 1000 in compressible flows, in Proceedings of AIAA Aerospace Sciences Meeting (American Institute of Aeronautics and Astronautics, Inc., Reston, VA, 2018), p. 0381.

[37] V. M. Boiko, V. P. Kiselev, S. P. Kiselev, A. N. Papyrin, S. V. Poplavsky, and V. M. Fomin, Shock wave interaction with a cloud of particles, Shock Waves 7, 275 (1997). 
[38] G. Emanuel and J. P. Jones, Compressible flow through a porous plate, Int. J. Heat Mass Transf. 11, 827 (1968).

[39] C. T. Crowe, J. D. Schwarzkopf, M. Sommerfeld, and Y. Tsuji, Multiphase Flows with Droplets and Particles (CRC Press, Boca Raton, FL, 2011).

[40] J. D. Schwarzkopf and J. A. Horwitz, BHR equations re-derived with immiscible particle effects, technical report, Los Alamos National Laboratory (2015).

[41] G. A. Brès, F. E. Ham, J. W. Nichols, and S. K. Lele, Unstructured large-eddy simulations of supersonic jets, AIAA J. 55, 1164 (2017).

[42] G. A. Bres, S. T. Bose, M. Emory, F. E. Ham, O. T. Schmidt, G. Rigas, and T. Colonius, Large-eddy simulations of co-annular turbulent jet using a Voronoi-based mesh generation framework, in Proceedings of AIAA/CEAS Aeroacoustics Conference (American Institute of Aeronautics and Astronautics, Inc., Reston, VA, 2018), p. 3302.

[43] M. Masquelet, J. Yan, A. Dord, G. Laskowski, L. Shunn, L. Jofre, and G. Iaccarino, Uncertainty quantification in large eddy simulations of a rich-dome aviation gas turbine, in ASME Turbo Expo 2017: Turbomachinery Technical Conference and Exposition (American Society of Mechanical Engineers, New York, 2017), pp. V04BT04A049-V04BT04A049.

[44] A. W. Vreman, An eddy-viscosity subgrid-scale model for turbulent shear flow: Algebraic theory and applications, Phys. Fluids 16, 3670 (2004).

[45] A. N. Osnes, M. Vartdal, M. G. Omang, and B. A. P. Reif, Numerical investigation of shock wave particle cloud interaction in cylindrical geometries, in Proceedings of the 10th National Conference on Computational Mechanics MekIT'19, edited by B. Skallerud and H. I. Andersson (International Center for Numerical Methods in Engineering, Barcelona, Spain, 2019).

[46] M. Sun, T. Saito, K. Takayama, and H. Tanno, Unsteady drag on a sphere by shock wave loading, Shock Waves 14, 3 (2005).

[47] I. Rodríguez, O. Lehmkuhl, R. Borrell, and A. Oliva, Flow dynamics in the turbulent wake of a sphere at sub-critical Reynolds numbers, Comput. Fluids 80, 233 (2013).

[48] G. Akiki, T. L. Jackson, and S. Balachandar, Pairwise interaction extended point-particle model for a random array of monodisperse spheres, J. Fluid Mech. 813, 882 (2017).

[49] R. Clift and W. H. Gauvin, Motion of particles in turbulent gas streams, in Proceedings of Chemchea (1970), pp. 14-28.

[50] E. Achenbach, The effects of surface roughness and tunnel blockage on the flow past spheres, J. Fluid Mech. 65, 113 (1974).

[51] T. Akutsu, Wall confinement effects for spheres in the Reynolds number range of 30-2000, PhD. thesis, University of British Columbia, 1977.

[52] S. Krishnan and A. Kaman, Effect of blockage ratio on drag and heat transfer from a centrally located sphere in pipe flow, Eng. Applicat. Computat. Fluid Mech. 4, 396 (2010).

[53] W. W. H. Yeung, On pressure invariance, wake width and drag prediction of a bluff body in confined flow, J. Fluid Mech. 622, 321 (2009). 